ALEA, Lat. Am. J. Probab. Math. Stat. 13, 481-519 (2016)

DOI: 10.30757/ALEA.v13-20

\title{
Functional Central Limit Theorem for the Interface of the Symmetric Multitype Contact Process
}

\section{Thomas Mountford and Daniel Valesin}

Ecole Polytechnique Fédérale de Lausanne

EPFL SB MATHAA PRST MA B1 517,

Station 8,

CH-1015 Lausanne, Switzerland

E-mail address: thomas.mountford@epfl.ch

$U R L:$ https://people.epfl.ch/thomas.mountford

University of Groningen

Nijenborgh 9,

9747AG Groningen, The Netherlands

E-mail address: d.rodrigues.valesin@rug.nl

URL: http://www.math.rug.nl/ valesin/

\begin{abstract}
We study the interface of the symmetric multitype contact process on $\mathbb{Z}$. In this process, each site of $\mathbb{Z}$ is either empty or occupied by an individual of one of two species. Each individual dies with rate 1 and attempts to give birth with rate $2 R \lambda$; the position for the possible new individual is chosen uniformly at random within distance $R$ of the parent, and the birth is suppressed if this position is already occupied. We consider the process started from the configuration in which all sites to the left of the origin are occupied by one of the species and all sites to the right of the origin by the other species, and study the evolution of the region of interface between the two species. We prove that, under diffusive scaling, the position of the interface converges to Brownian motion.
\end{abstract}

\section{Introduction}

The multitype contact process is a stochastic process that can be seen as a model for the evolution of different biological species competing for the occupation of space. It was introduced in Neuhauser (1992) as a modification of Harris' (singletype) contact process (Harris, 1974).

Let us give the definition of the multitype contact process $\left(\xi_{t}\right)_{t \geq 0}$ on $\mathbb{Z}^{d}$ with (at most) two types. We will need the parameters: $R_{1}, R_{2} \in \mathbb{N}^{*}$ and $\bar{\delta}_{1}, \delta_{2}, \lambda_{1}, \lambda_{2}>0$.

Received by the editors September 21, 2015; accepted May 9, 2016.

2010 Mathematics Subject Classification. 60K35, 82C22.

Key words and phrases. Interacting particle systems, contact process, interface. 
$\left(\xi_{t}\right)_{t \geq 0}$ is then the Markov process with state space $\{0,1,2\}^{\mathbb{Z}^{d}}$ and generator given by $\mathcal{L}=\mathcal{L}_{1}+\mathcal{L}_{2}$, with

$$
\left(\mathcal{L}_{i} f\right)(\xi)=\sum_{\substack{x \in \mathbb{Z}^{d}: \\
\xi(x)=i}} \delta_{i} \cdot\left[f\left(\xi^{0 \rightarrow x}\right)-f(\xi)\right]+\sum_{\substack{x \in \mathbb{Z}^{d}: \\
\xi(x)=0}} \sum_{\begin{array}{c}
y \in \mathbb{Z}^{d}: \\
|x-y| \leq R_{i}, \\
\xi(y)=i
\end{array}} \lambda_{i} \cdot\left[f\left(\xi^{i \rightarrow x}\right)-f(\xi)\right], \quad i=1,2,
$$

where $f:\{0,1,2\}^{\mathbb{Z}^{d}} \rightarrow \mathbb{R}$ is a function that depends only on finitely many coordinates, $|\cdot|$ is the $\ell^{\infty}$ norm and

$$
\xi^{i \rightarrow x}(y)=\left\{\begin{array}{ll}
\xi(y) & \text { if } y \neq x \\
i & \text { if } y=x
\end{array} \quad i=0,1,2\right.
$$

We will adopt throughout the paper the following terminology: vertices are called sites, sites in state 0,1 and 2 are respectively said to be empty or to have a type 1 or type 2 occupant (or individual), and elements of $\{0,1,2\} \mathbb{Z}^{d}$ are called configurations. Additionally, $\delta_{1}, \delta_{2}$ are called death rates, $R_{1}, R_{2}$ are ranges and $\lambda_{1}, \lambda_{2}$ are birth rates (or sometimes infection rates).

Let us now explain the dynamics in words. Two kinds of transitions can occur. First, an individual of type $i$ dies with rate $\delta_{i}$, leaving its site empty. Second, given a pair of sites $x, y$ with $|x-y| \leq R_{i}, \xi(x)=i$ (with $i=1$ or 2 ) and $\xi(y)=0$, the occupant of $x$ gives birth at $y$ with rate $\lambda_{i}$, so that a new individual of type $i$ is placed at $y$. Note that, under these rules, births only occur at empty sites, so that the state of a site can never change directly from 1 to 2 or from 2 to 1 .

In case only one type (say, type 1 ) is present, this reduces to the contact process introduced in Harris (1974), to be denoted here by $\left(\zeta_{t}\right)_{t \geq 0}$ in order to distinguish it from the multitype version. We refer the reader to Liggett (1999) for an exposition of the contact process and the statements about it that we will gather in this Introduction and in Section 2.

Let $\left(\zeta_{t}^{\{0\}}\right)_{t \geq 0}$ be the (one-type) contact process with rates $\delta_{1}=\delta=1, \lambda_{1}=\lambda>$ $0, R_{1}=R \in \mathbb{Z}_{+}^{*}$ and the initial configuration in which only the origin is occupied. Denote by $\underline{0}$ the configuration in which every vertex is empty, and note that this is a trap state for the dynamics. There exists $\lambda_{c}=\lambda_{c}\left(\mathbb{Z}^{d}, R\right)$ (depending on the dimension $d$ and the range $R$ ) such that

$$
\mathbb{P}\left[\text { there exists } t>0 \text { such that } \zeta_{t}^{\{0\}}=\underline{0}\right]=1 \quad \text { if and only if } \lambda \leq \lambda_{c} .
$$

This phase transition is the most fundamental property of the contact process. The process is called subcritical, critical and supercritical respectively in the cases $\lambda<\lambda_{c}, \lambda=\lambda_{c}$ and $\lambda>\lambda_{c}$.

In this paper, we will consider the multitype contact process $\left(\xi_{t}\right)$ on $\mathbb{Z}$ with parameters

$$
\delta_{1}=\delta_{2}=1, \quad R_{1}=R_{2}=R, \quad \lambda_{1}=\lambda_{2}=\lambda>\lambda_{c}(\mathbb{Z}, R) .
$$

We emphasize that the quantity $\lambda_{c}(\mathbb{Z}, R)$ that appears here is the one associated to the one-type process, as in (1.3). We will be particularly interested in the 'heaviside' initial configuration,

$$
\xi_{0}^{h}(x)= \begin{cases}1 & \text { if } x \leq 0 \\ 2 & \text { if } x>0\end{cases}
$$


We will denote by $\left(\xi_{t}^{h}\right)_{t \geq 0}$ the process with parameters (1.4) and initial configuration $\xi_{0}^{h}$. We let

$$
r_{t}=\sup \left\{x: \xi_{t}^{h}(x)=1\right\}, \quad \ell_{t}=\inf \left\{x: \xi_{t}^{h}(x)=2\right\}, \quad i_{t}=\left(r_{t}+\ell_{t}\right) / 2 .
$$

The interval delimited by $r_{t}$ and $\ell_{t}$ is called the interface at time $t$, and $i_{t}$ is the position of the interface at time $t$. The choice of the middle point of the interval as the position of the interface is somewhat arbitrary and will not matter for all the results obtained in this paper.

In case $R=1$, it follows readily from inspecting the generator in (1.1) that $r_{t}<\ell_{t}$ for all $t$. If $R>1$, both $r_{t}<\ell_{t}$ and $r_{t}>\ell_{t}$ are possible (in the latter case we say that we have a positive interface, and in the previous case, a negative interface). In Valesin (2010), it is shown that the process $\left(\left|r_{t}-\ell_{t}\right|\right)_{t \geq 0}$, which describes the evolution of the size of the interface, is stochastically tight:

Theorem 1.1. Valesin (2010) If $R \in \mathbb{Z}_{+}^{*}$ and $\lambda>\lambda_{c}(\mathbb{Z}, R)$, then

for any $\varepsilon>0$ there exists $L>0$ such that $\mathbb{P}\left[\left|r_{t}-\ell_{t}\right|>L\right]<\varepsilon$ for all $t \geq 0$.

In the present paper, we will continue the study of the interface, but we will focus on its position rather than its size. Our main result is

Theorem 1.2. If $R \in \mathbb{Z}_{+}^{*}$ and $\lambda>\lambda_{c}(\mathbb{Z}, R)$, then there exists $\sigma>0$ such that

$$
\left(t^{-1 / 2} \cdot i_{s t}\right)_{s \geq 0} \underset{\text { (dist. })}{\stackrel{t \rightarrow \infty}{\longrightarrow}}\left(B_{s}\right)_{s \geq 0},
$$

where $\left(B_{s}\right)_{s>0}$ denotes Brownian motion with diffusive constant $\sigma$, and convergence holds in the space $D=D[0, \infty)$ of càdlàg trajectories with the Skorohod topology.

Our proof of this result follows the usual two steps: verifying convergence of finite-dimensional distributions and tightness of trajectories in $D$ (see Section 16 of Billingsley, 1999). We thus prove the following propositions, both applicable to the case $R \in \mathbb{Z}_{+}^{*}$ and $\lambda>\lambda_{c}(\mathbb{Z}, R)$ :

Proposition 1.3. There exists $\sigma>0$ such that, for any $0=a_{0}<a_{1}<\cdots<a_{k}$ we have

$$
t^{-1 / 2} \cdot\left(i_{a_{1} \cdot t}, i_{a_{2} \cdot t}-i_{a_{1} \cdot t}, \ldots, i_{a_{k} \cdot t}-i_{a_{k-1} \cdot t}\right) \underset{(\text { dist. })}{\stackrel{t \rightarrow \infty}{\longrightarrow}}\left(N_{1}, \ldots, N_{k}\right),
$$

where $N_{1}, \ldots, N_{k}$ are independent and $N_{j} \sim \mathcal{N}\left(0, \sigma^{2}\left(a_{j}-a_{j-1}\right)\right)$.

Proposition 1.4. For any $\varepsilon>0$ there exists a compact set $K \subset D$ such that

$$
\liminf _{t \rightarrow \infty} \mathbb{P}\left[\left(t^{-1 / 2} \cdot i_{s t}\right)_{s \geq 0} \in K\right]>1-\varepsilon .
$$

In proving these propositions, we will establish a result of independent interest, which we call interface regeneration. We will explain it here only informally; the precise result depends on a few definitions and is given in Theorem 2.14. Given $s>0$, consider the configuration $\xi_{s}^{h}$ and assume the interface position $i_{s}=x$. Suppose we define a new configuration $\bar{\xi}$ by putting 1's in all sites to the left of $i_{s}$ and 2's to the right of $i_{s}$. We then show that it is possible to construct, in the same probability space as that of $\left(\xi_{t}\right)_{t>0}$, a multitype contact process started from time $s,\left(\xi_{t}^{\prime}\right)_{t \geq s}$, such that $\xi_{s}^{\prime}=\bar{\xi}$ and moreover, the interface positions for $\left(\xi_{t}\right)$ and for $\left(\xi_{t}^{\prime}\right)$ are never too far from each other. Since the evolution of the interface of $\xi^{\prime}$ 
has the same distribution as that of the original process (except for a space-time shift), this regeneration allows us to argue that, if we consider large time intervals $I_{1}, I_{2}, \ldots$, then the displacement of $i_{s}$ in each interval follows approximately the same law.

In many of our proofs, we study the time dual of the multitype contact process. This dual, called the ancestor process, was first considered in Neuhauser (1992) and further studied in Valesin (2010). In these references, it was shown that the ancestor process behaves approximately as a system of coalescing random walks on $\mathbb{Z}$. Because of this, our proofs of Propositions 1.3 and 1.4 are inspired by arguments that apply to coalescing random walks and the voter model, an interacting particle system whose dual is (exactly) equal to coalescing random walks. In particular, a key estimate for the proof of Proposition 1.4 (see Lemma 4.3) was inspired in an argument by Sun for coalescing random walks (Sun, 2005).

\section{Background on the contact process}

2.1. Notation on sets and configurations. Given a set $A$, we denote by $\# A$ its cardinality and by $\mathbb{1}_{A}$ its indicator function.

We will reserve the letter $\zeta$ to denote elements of $\{0,1\}^{\mathbb{Z}}$, as well as the onetype contact process, and the letter $\xi$ for elements of $\{0,1,2\}^{\mathbb{Z}}$ and the multitype process. We denote by $\underline{0}$ the configuration in which every vertex is in state 0 . We write $\{\xi=i\}=\{x \in \mathbb{Z}: \xi(x)=i\}$ (and similarly for $\zeta$ ). Given $A \subset \mathbb{Z}, " \xi \equiv i$ on $A$ " means that $\xi(x)=i$ for all $x \in A$ (and similarly for $\zeta$ ).

Throughout the paper, we fix the parameters $R \in \mathbb{Z}_{+}^{*}$ and $\lambda>\lambda_{c}(R, \mathbb{Z})$. All the processes we will consider will be defined from these two parameters.

2.2. One-type contact process. We will now briefly survey some background material on the (one-type) contact process.

a) Graphical construction and infection paths. A graphical construction or Harris system is a family of independent Poisson processes on $[0, \infty)$,

$$
H=\left(\left(D^{x}\right)_{x \in \mathbb{Z}},\left(D^{x, y}\right)_{x, y \in \mathbb{Z}, 0<|x-y| \leq R}\right), \quad \begin{array}{ll}
\text { each } D^{x} \text { with rate } 1, \\
\text { each } D^{x, y} \text { with rate } \lambda .
\end{array}
$$

We view each of these processes as a random discrete subset of $[0, \infty)$. An arrival at time $t$ of the process $D^{x}$ is called a recovery mark at $x$ at time $t$, and an arrival at time $t$ of the process $D^{x, y}$ is called an arrow or transmission from $x$ to $y$ at time $t$. This terminology is based on the usual interpretation that is given to the contact process, namely: vertices are individuals, individuals in state 1 are infected and individuals in state 0 are healthy. Although we will focus mostly on the multitype contact process, which we see as a model for competition rather than the spread of an infection, we will still use some infection-related terminology that comes from the study of the classical process.

We will sometimes need to consider restrictions of $H$ to time intervals, and also translations of $H$. We therefore introduce the following notation, for $t>0$ and 
$z \in \mathbb{Z}:$

$$
\begin{aligned}
& D_{[0, t]}^{x}=D^{x} \cap[0, t], \quad D^{x} \circ \theta(z, t)=\left\{s-t: s \in D^{x-z}, s \geq t\right\}, \\
& D_{[0, t]}^{x, y}=D^{x, y} \cap[0, t], \quad D^{x, y} \circ \theta(z, t)=\left\{s-t: s \in D^{x-z, y-z}, s \geq t\right\}, \\
& H_{[0, t]}=\left(\left(D_{[0, t]}^{x}\right)_{x \in \mathbb{Z}},\left(D_{[0, t]}^{(x, y)}\right)_{x, y \in \mathbb{Z}^{d}, 0<|x-y| \leq R}\right), \\
& H \circ \theta(z, t)=\left(\left(D^{x} \circ \theta(z, t)\right)_{x \in \mathbb{Z}},\left(D^{(x, y)} \circ \theta(z, t)\right)_{x, y \in \mathbb{Z}^{d}, 0<|x-y| \leq R}\right) .
\end{aligned}
$$

Given a (deterministic or random) initial configuration $\zeta_{0}$ and a Harris system $H$, it is possible to construct the contact process $\left(\zeta_{t}\right)_{t \geq 0}$ started from $\zeta_{0}$ by applying the following rules to the arrivals of the Poisson processes in $H$ :

a recovery mark at a site makes it healthy:

$$
\text { if } t \in D^{x} \text {, then } \zeta_{t}=\zeta_{t-}^{0 \rightarrow x} \text {; }
$$

an arrow with infected starting point makes the end point infected:

$$
\text { if } t \in D^{x, y} \text { and } \zeta_{t-}(x)=1 \text {, then } \zeta_{t}=\zeta_{t-}^{1 \rightarrow y}
$$

(recall that $\zeta^{i \rightarrow x}$ was defined in (1.2)). That this can be done in a consistent manner, and that it yields a Markov process with the desired infinitesimal generator, is a non-trivial result which (as the other statements in this section) the reader can find in Liggett (1999).

Given $x, y \in \mathbb{Z}, t^{\prime}>t \geq 0$ and a Harris system $H$, an infection path in $H$ from $(x, t)$ to $\left(y, t^{\prime}\right)$ is a càdlàg path $\gamma:\left[t, t^{\prime}\right] \rightarrow \mathbb{Z}$ such that

$$
\begin{aligned}
& \gamma(t)=x, \quad \gamma\left(t^{\prime}\right)=y, \\
& s \notin D^{\gamma(s)} \text { for all } s \in\left[t, t^{\prime}\right] \text { and } s \in D^{\gamma(s-), \gamma(s)} \text { whenever } \gamma(s-) \neq \gamma(s)
\end{aligned}
$$

In words, $\gamma$ may not touch recovery marks and $\gamma$ can only jump from site $x$ to site $y$ at time $s$ if there is an arrow from $x$ to $y$ at $s$. We employ the following notation. In case there is an infection path from $(x, t)$ to $\left(y, t^{\prime}\right)$, we write $(x, t) \leftrightarrow\left(y, t^{\prime}\right)$ in $H$ (or simply $(x, t) \leftrightarrow\left(y, t^{\prime}\right)$ if $H$ is clear from the context). Given sets $A, B \subset \mathbb{Z}$, and $I_{1}, I_{2} \subset[0, \infty)$, we write $A \times I_{1} \leftrightarrow B \times I_{2}$ if $(x, t) \leftrightarrow\left(y, t^{\prime}\right)$ for some $(x, t) \in A \times I_{1}$ and $\left(y, t^{\prime}\right) \in B \times I_{2}$. We will also write $A \times I_{1} \leftrightarrow\left(y, t^{\prime}\right)$ if $(x, t) \leftrightarrow\left(y, t^{\prime}\right)$ for some $(x, t) \in A \times I_{1}$, and similarly $(x, t) \leftrightarrow B \times I_{2}$. Finally, we adopt the convention to put $(x, t) \leftrightarrow(x, t)$.

When $\left(\zeta_{t}\right)$ is constructed with the rules (2.3) and (2.4), for any $(x, t) \in \mathbb{Z} \times[0, \infty)$, $x$ is infected at time $t$ if and only if there exists a site $y \in \mathbb{Z}$ which is infected at time 0 and so that an infection path connects $(y, 0)$ and $(x, t)$ :

$$
\zeta_{t}(x)=\mathbb{1}_{\left\{\left\{\zeta_{0}=1\right\} \times\{0\} \leftrightarrow(x, t)\right\}} \text { for all } x \in \mathbb{Z}, t \geq 0 .
$$

We will always assume that the contact process is constructed from a Harris system (this will also be the case for the multitype contact process, which, as we will explain shortly, can be constructed from the same $H$ as the one given above). Additionally, we will often consider more than one process at a given time, and implicitly assume that all the processes are built in the same probability space, using the same $H$.

Let us now list a few facts and estimates that we will need. 
b.) Basic facts about the distribution of Harris systems. Given $A \subset \mathbb{Z}$, $x \in \mathbb{Z}$ and $t \geq 0$, by invariance of the law of Poisson processes with respect to time reversal, the following holds:

$$
\begin{aligned}
\mathbb{P}\left[\zeta_{t}^{A}(x)=1\right] & =\mathbb{P}[A \times\{0\} \leftrightarrow(x, t)] \\
& =\mathbb{P}[(x, 0) \leftrightarrow A \times\{t\}]=\mathbb{P}\left[\zeta_{t}^{x}(y)=1 \text { for some } y \in A\right] .
\end{aligned}
$$

This is a particularly simple instance of the well-known self-duality property of the contact process.

Given $A \subset \mathbb{Z}$ and $t \geq 0$, due to invariance of the law of the Harris system with respect to space-time translations, the processes

$$
\left(\zeta_{s-t}^{A}\right)_{s \geq t} \quad \text { and } \quad\left(\mathbb{1}_{\{x \in \mathbb{Z}: A \times\{t\} \leftrightarrow(x, s)\}}\right)_{s \geq t}
$$

have the same distribution. We refer to the latter as the contact process started at time $t$ with $A$ occupied.

By a simple comparison with a Poisson process, we can show that there exist $\kappa, c>0$ (depending on $\lambda$ and $R$ ) such that

$$
\mathbb{P}\left[(x, r) \leftrightarrow[x-\kappa t, x+\kappa t]^{c} \times\{r+t\}\right] \leq e^{-c t} \text { for any } x \in \mathbb{Z}, r \geq 0, t>0 .
$$

c.) Extinction time. Given $A \subset \mathbb{Z}$ and $t \geq 0$, define the extinction time of the contact process started at time $t$ with $A$ occupied as the infimum of the times that cannot be reached by infection paths started from $A \times\{t\}$ :

$$
T^{A \times\{t\}}=\inf \left\{t^{\prime} \geq t: A \times\{t\} \leftrightarrow \mathbb{Z} \times\left\{t^{\prime}\right\}\right\} .
$$

Note that this is a stopping time with respect to the natural filtration of Harris systems. We write $T^{(x, t)}$ instead of $T^{\{x\} \times\{t\}}$ and, in case $t=0$, we omit it and write $T^{A}$ and $T^{x}$. Note that the distribution of $T^{A \times\{t\}}$ is equal to that of $t+T^{A \times\{0\}}$.

By (1.3) and the assumption that $\lambda>\lambda_{c}$,

$$
\mathbb{P}\left[T^{0}=\infty\right]>0 .
$$

In case $T^{A \times\{t\}}=\infty$, we write $A \times\{t\} \leftrightarrow \infty$. Similarly, when $T^{(x, t)}=\infty$, we write $(x, t) \leftrightarrow \infty$.

In Theorem 2.30 in Liggett (1999), we find that there exists $c>0$ (depending on $R$ and $\lambda$ ) such that

$$
\mathbb{P}\left[t<T^{0}<\infty\right] \leq e^{-c t} \text { for any } t>0
$$

and

$$
\mathbb{P}\left[T^{A}<\infty\right]<e^{-c \# A} \text { for any } A \subset \mathbb{Z}, A \neq \varnothing .
$$

In the mentioned theorem, these estimates are obtained for the case $R=1$, but the method of proof is a comparison with oriented percolation that works equally well for $R>1$.

d.) An insulation result and an application. The following result deals with the contact process started from an initial configuration $\zeta_{0}$ in which an interval $[a, b]$ has the property that, in the two (large) intervals $[a-S, a)$ and $(b, b+S]$, all vertices are occupied (we think of these large intervals as "insulating" $[a, b]$ from the outside). It then guarantees that, in a linearly growing spacial set which contains $[a, b]$ at time zero, the process is likely to coincide with the process started from $\zeta_{0}^{\prime}$, the modification of $\zeta_{0}$ in which all sites outside $[a, b]$ are set to occupied. 
Lemma 2.1. There exists $\bar{\beta}>0$ such that the following holds. For any $\varepsilon>0$ there exists $S_{0}>0$ such that, if $S \geq S_{0}$ and $\zeta_{0}$, $\zeta_{0}^{\prime}$ satisfy, for some $a<b$,

$$
\begin{aligned}
& \zeta_{0}(x)=\zeta_{0}^{\prime}(x) \text { for all } x \in[a, b] ; \\
& \zeta_{0}(x)=1 \text { for all } x \in[a-S, a) \cup(b, b+S] ; \\
& \zeta_{0}^{\prime}(x)=1 \text { for all } x \in(-\infty, a) \cup(b, \infty)
\end{aligned}
$$

and $\left(\zeta_{t}\right),\left(\zeta_{t}^{\prime}\right)$ are contact processes started from $\zeta_{0}$ and $\zeta_{0}^{\prime}$ and constructed with the same Harris system, then, with probability larger than $1-\varepsilon$,

$$
\zeta_{t}(x)=\zeta_{t}^{\prime}(x) \text { for all } x \in[a-\bar{\beta} t, b+\bar{\beta} t] \text { and } t \geq 0 .
$$

This result is easy to prove when the process only has nearest-neighbor interactions, $R=1$, and much harder for general $R$. The proof (for any $R \in \mathbb{N}^{*}$ ) follows from Proposition 2.7 in Andjel et al. (2010) (see also the treatment of the event $\mathcal{H}_{2}$ in page 11 of that paper). The key idea is an event which the authors called the formation of a descendancy barrier; this means that in a space-time set of the form $\mathcal{C}_{x}=\{[x-\bar{\beta} t, x+\bar{\beta} t]: t \geq 0\}$, every vertex that is reachable by an infection path from $\mathbb{Z} \times\{0\}$ is reachable by an infection path from $(x, 0)$. For the statement of the present lemma, it would suffice to argue that, if $S$ is large, with high probability one can find $x \in[a-S, a)$ and $y \in(b, b+S]$ so that a descendancy barrier is formed from both $x$ and $y$.

Remark 2.2. The above lemma also holds, with the same proof, for $a=-\infty$ or $b=\infty$.

We will need the following consequence of Lemma 2.1.

Lemma 2.3. For any $\varepsilon>0$ and $\sigma, \sigma^{\prime}$ with $-\bar{\beta} \leq \sigma<\sigma^{\prime} \leq \bar{\beta}$ there exists $S_{0}>0$ such that, if $S \geq S_{0}$ and $\zeta_{0}(x)=1$ for all $x \in[-S, S]$, then with probability larger than $1-\varepsilon$,

$$
\text { for all } t \geq 0 \text {, there exists } x \in\left[-S+\sigma t, S+\sigma^{\prime} t\right] \text { such that } \zeta_{t}(x)=1 .
$$

Proof: By Lemma 2.1, it suffices to prove that, given $\varepsilon, \sigma, \sigma^{\prime}$, there exists $S_{0}$ so that, for $S \geq S_{0}$,

$\mathbb{P}\left[\right.$ for all $t \geq 0$, there exists $x \in\left[-S+\sigma t, S+\sigma^{\prime} t\right]$ with $\left.\zeta_{t}^{\mathbb{Z}}(x)=1\right]>1-\varepsilon$,

where $\left(\zeta_{t}^{\mathbb{Z}}\right)$ denotes the one-type contact process started from full occupancy. For any $t$ we have

$$
\begin{aligned}
& \mathbb{P}\left[\zeta_{t}^{\mathbb{Z}} \equiv 0 \text { on }\left[-S+\sigma t, S+\sigma^{\prime} t\right]\right] \\
& =\mathbb{P}\left[\mathbb{Z} \times\{0\} \leftrightarrow(x, t) \text { for all } x \in\left[-S+\sigma t, S+\sigma^{\prime} t\right]\right] \\
& \stackrel{(2.7)}{=} \mathbb{P}\left[(x, 0) \leftrightarrow \mathbb{Z} \times\{t\} \text { for all } x \in\left[-S+\sigma t, S+\sigma^{\prime} t\right]\right] \\
& \leq \mathbb{P}\left[T^{\left[-S+\sigma t, S+\sigma^{\prime} t\right]}<\infty\right] \stackrel{(2.10)}{<} e^{-2 c S-c\left(\sigma^{\prime}-\sigma\right) t} .
\end{aligned}
$$


Also, using the Strong Markov Property it is easy to verify that, for some $\delta>0$ that depends on $\sigma, \sigma^{\prime}, \lambda$ and $R$ but not on $t$,

$$
\begin{aligned}
\mathbb{P}\left[\zeta_{t}^{\mathbb{Z}} \equiv 0 \text { on }\left[-S+\sigma t, S+\sigma^{\prime} t\right]\right. \\
\qquad \delta \cdot \mathbb{P}\left[\zeta_{s}^{\mathbb{Z}} \equiv 0 \text { on }\left[-S+\sigma s, S+\sigma^{\prime} s\right] \text { for some } s \in[t-1, t]\right]
\end{aligned}
$$

for all $t \geq 1$. In conclusion,

$$
\begin{aligned}
& \mathbb{P}\left[\text { there exists } t>0: \zeta_{t}^{\mathbb{Z}} \equiv 0 \text { on }[-S+\sigma t, S+\sigma t]\right] \\
& \leq \sum_{t=1}^{\infty} \mathbb{P}\left[\text { there exists } s \in[t-1, t]: \zeta_{t}^{\mathbb{Z}} \equiv 0 \text { on }\left[-S+\sigma s, S+\sigma^{\prime} s\right]\right] \\
& \leq \frac{1}{\delta} \sum_{t=1}^{\infty} e^{-2 c S-c\left(\sigma^{\prime}-\sigma\right) t}
\end{aligned}
$$

which is smaller than $\varepsilon$ if $S$ is large enough, since $\sigma^{\prime}-\sigma>0$.

e.) Invasion-free infection paths. When we construct the contact process $\left(\zeta_{t}^{A}\right)_{t \geq 0}$, arrows in the Harris systems which land on space-time points which were already in state 1 have no effect. The following result states that, if $A \times\{0\} \leftrightarrow(x, t)$, then we can find exactly one infection path from $A \times\{0\}$ to $(x, t)$ in which all the jumps correspond to arrows that land on empty sites. We call such an infection path invasion-free; we stress that this property depends on the initial occupancy $A$, since the set of space-time points which are occupied depends on $A$. Invasion-free infection paths will be very useful for the graphical construction of the multitype contact process.

Lemma 2.4. Let $H$ be a Harris system, $A \subset \mathbb{Z}$ and $(x, t) \in \mathbb{Z} \times(0, \infty)$. Then, either $A \times\{0\} \leftrightarrow(x, t)$ or there exists a unique infection path $\gamma:[0, t] \rightarrow \mathbb{Z}$ such that $\gamma(0) \in A, \gamma(t)=x$ and every jump of $\gamma$ lands on a site that is empty in $\left(\zeta_{s}^{A}\right)_{s \geq 0}$, that is,

$$
\gamma(s) \neq \gamma(s-) \Longrightarrow \zeta_{s-}^{A}(\gamma(s))=0, \quad s \in[0, t] .
$$

Proof: Assume $A \times\{0\} \leftrightarrow(x, t)$. For every infection path $\gamma$ from $A \times\{0\}$ to $(x, t)$, we define $f(\gamma)$ as follows. If $\gamma$ satisfies (2.13), we let $f(\gamma)=-\infty$. Otherwise, we let $f(\gamma)$ be the last jump time of $\gamma$ which violates (2.13), that is,

$$
f(\gamma)=\max \left\{s: \gamma(s) \neq \gamma(s-), \zeta_{s-}^{A}(\gamma(s))=1 .\right.
$$

If we have $f(\gamma)=s^{*} \neq-\infty$, then there exists an infection path $\gamma^{\prime}$ from $A \times\{0\}$ to $\left(\gamma\left(s^{*}\right), s^{*}\right)$ whose last jump occurs before time $s^{*}$. Defining a path $\hat{\gamma}$ which is equal to $\gamma^{\prime}$ in $\left[0, s^{*}\right]$ and equal to $\gamma$ in $\left[s^{*}, t\right]$, we then obtain $f(\hat{\gamma})<s^{*}=f(\gamma)$.

In order to obtain a path satisfying (2.13), we can start with an arbitrary infection path $\gamma$ from $A \times\{0\}$ to $(x, t)$ and apply the above procedure recursively (that is, letting $\gamma^{(1)}=\hat{\gamma}, \gamma^{(2)}=\widehat{\gamma^{(1)}}$ etc.) until $f$ reaches $-\infty$. To see that the recursion must indeed reach a path for which $f$ is $-\infty$, note that the value of $f$ decreases with each iteration, and moreover $f$ can only attain finitely many values, as there are almost surely only finitely many infection paths reaching $(x, t)$.

The uniqueness statement is evident: if $\gamma_{1}$ and $\gamma_{2}$ are two distinct infection paths from $A \times\{0\}$ to $(x, t)$, then at some point either $\gamma_{1}$ jumps to a space-time point occupied by $\gamma_{2}$ or vice-versa, so (2.13) cannot be satisfied by both paths. 

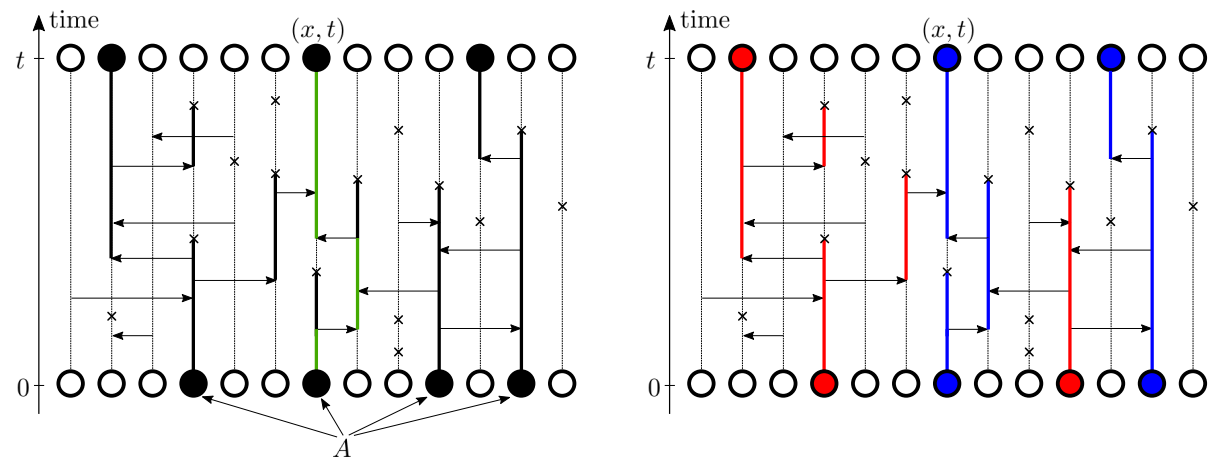

Figure 2.1. Left: illustration of Lemma 2.4. Three infection paths can be found from $A \times\{0\}$ to $(x, t)$; the unique one in which all jumps land on empty space-time points appears in green. Right: multitype contact process built with the same Harris system as the one on the left. The two types are represented by the colors blue and red. Note that the infection path that appears in green on the left picture is entirely blue here.

\subsection{Multitype contact process.}

a.) Graphical construction and first properties. Due to our symmetric choice of parameters in (1.4), it is possible to construct the multitype contact process with the same graphical construction $H$ as the one we have given in (2.1) for the singletype process. The effects of recovery marks and arrows are:

a recovery mark at a site kills its occupant:

$$
\text { if } t \in D^{x} \text {, then } \xi_{t}=\xi_{t-}^{0 \rightarrow x} \text {; }
$$

an arrow with a type $i$ occupant at the starting point creates a new type $i$

occupant at the end point, provided the end point was empty:

$$
\text { if } t \in D^{x, y}, \xi_{t-}(x)=i \text { and } \xi_{t-}(y)=0 \text {, then } \xi_{t}=\xi_{t-}^{i \rightarrow y}, \quad i=1,2 \text {, }
$$

where $\xi^{i \rightarrow x}$ is defined in (1.2). These rules lead to the correct transition rates, as prescribed in (1.1) and (1.4). Figure 2.1 illustrates the construction.

This picture also illustrates an important fact: if we only distinguish empty sites from occupied sites (in the picture, if we treat blue and red as a single color), we re-obtain the single-type process. Formally,

$$
\begin{array}{r}
\left(\mathbb{1}_{\left\{\xi_{t}(x) \neq 0\right\}}: x \in \mathbb{Z}\right)_{t \geq 0} \text { has same distribution as a one-type contact } \\
\text { process started from }\left(\mathbb{1}_{\left\{\xi_{0}(x) \neq 0\right\}}: x \in \mathbb{Z}\right) .
\end{array}
$$

To verify that this holds, note that the rules (2.14) and (2.15) reduce to (2.3) and (2.4) when the two types are treated as a single type. A consequence of (2.16) is that we can still use infection paths to decide whether a space-time point is empty or occupied:

$$
\xi_{t}(x) \neq 0 \text { if and only if }\left\{y: \xi_{0}(y) \neq 0\right\} \times\{0\} \leftrightarrow(x, t) .
$$


This is quite convenient, but there is a drawback: in case $\xi_{t}(x) \neq 0$, it is not so simple to deduce its value from $\xi_{0}$ and the infection paths in $H$. This is where Lemma 2.4 comes into play: if an infection path $\gamma:[0, t] \rightarrow \mathbb{Z}$ is invasion-free and $\xi_{0}(\gamma(0))=i \in\{1,2\}$, then it manages to carry type $i$, that is, $\xi_{s}(\gamma(s))=i$ for all $s$. This is again illustrated in Figure 2.1 and summarized as follows:

Lemma 2.5. Let $\left(\xi_{t}\right)_{t \geq 0}$ be the multitype contact process started from a fixed $\xi_{0} \in$ $\{0,1,2\}^{\mathbb{Z}}$ and constructed with a Harris system. Then,

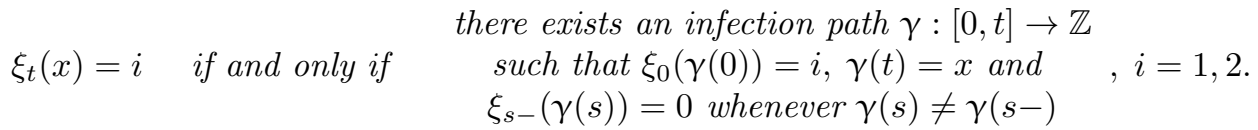

Moreover, for every $(x, t)$ there exists at most one infection path satisfying the stated properties.

As already noted, it will often be convenient to construct several processes, one-type or multitype or both, in the same probability space and using a single realization of $H$. When we do so, the following will be quite useful.

Claim 2.6. If $\left(\xi_{t}\right)_{t \geq 0},\left(\xi_{t}^{\prime}\right)_{t \geq 0}$ are constructed with the same Harris system,

$$
\begin{aligned}
& \left\{\xi_{0}=2\right\} \subseteq\left\{\xi_{0}^{\prime}=2\right\} \text { and }\left\{\xi_{0}=1\right\} \supseteq\left\{\xi_{0}^{\prime}=1\right\} \text { implies } \\
& \left\{\xi_{t}=2\right\} \subseteq\left\{\xi_{t}^{\prime}=2\right\} \text { and }\left\{\xi_{t}=1\right\} \supseteq\left\{\xi_{t}^{\prime}=1\right\} \text { for all } t .
\end{aligned}
$$

Proof: Simply consider the partial order

$$
\xi \prec \xi^{\prime} \quad \Leftrightarrow \quad\{\xi=2\} \subseteq\left\{\xi^{\prime}=2\right\} \text { and }\{\xi=1\} \supseteq\left\{\xi^{\prime}=1\right\}
$$

and note that the rules (2.14) and (2.15) preserve this order.

b.) Ancestor process. Given a multitype contact process $\left(\xi_{t}\right)_{t \geq 0}$ and $(x, t) \in$ $\mathbb{Z} \times[0, \infty)$ with $\xi_{t}(x)=i \in\{1,2\}$, Lemma 2.5 shows us how to locate the site $y$ which contained, at time 0 , the individual of type $i$ from which the occupant of $(x, t)$ descends. This suggests the definition of an ancestor process which holds a duality relation with the multitype contact process.

The ancestor process was first defined by Neuhauser (1992) in a more general version than the one we will present here. The definition in that paper depends on an algorithmic exploration of the Harris system, starting at $(x, t)$ and moving downwards to time 0 . Here we do not need to use Neuhauser's algorithm: our Lemmas 2.4 and 2.5 already allow us to define the ancestor process and obtain the results we will need.

Fix a Harris system $H$ and $x \in \mathbb{Z}$. We will define a process $\left(\eta_{t}^{x}\right)_{t \geq 0}$ on $\mathbb{Z} \cup\{\triangle\}$, where $\triangle$ represents a "cemetery" state. For each $t, \eta_{t}^{x}$ will be measurable with respect to $H_{[0, t]}$, the restriction of the Harris system to $[0, t]$, and will satisfy:

$$
\eta_{t}^{x} \in\left\{y: \zeta_{t}^{x}(y)=1\right\} \text { if } t<T^{x} \quad \text { and } \quad \eta_{t}^{x}=\triangle \text { if } t \geq T^{x} .
$$

For fixed $t>0$, we recall the notation introduced in (2.2) and define the reversed Harris system $\hat{H}_{[0, t]}$ by

$$
\begin{aligned}
& \hat{H}_{[0, t]}=\left(\left(\hat{D}_{[0, t]}^{x}\right)_{x \in \mathbb{Z}},\left(\hat{D}_{[0, t]}^{x, y}\right)_{x, y \in \mathbb{Z}, 0<|x-y| \leq R}\right), \text { where } \\
& \hat{D}_{[0, t]}^{x}=\left\{t-s: s \in D^{x} \cap[0, t]\right\} \text { and } \hat{D}_{[0, t]}^{x, y}=\left\{t-s: s \in D^{y, x} \cap[0, t]\right\} .
\end{aligned}
$$


In words, $\hat{H}_{[0, t]}$ is the Harris system on the time interval $[0, t]$ obtained from $H \cap[0, t]$ by reversing time and reversing the direction of the arrows (it is useful to think of $H_{[0, t]}$ and $\hat{H}_{[0, t]}$ drawn in the same picture, with the time direction of each being the opposite of that of the other).

Now, we define $\eta_{t}^{x}$ as follows:

- in case $\mathbb{Z} \times\{0\} \leftrightarrow(x, t)$ in $\hat{H}_{[0, t]}$, we let $\eta_{t}^{x}=\triangle$;

- otherwise, by Lemma 2.4 (with $A=\mathbb{Z}$ ), there exists a unique infection path $\gamma:[0, t] \rightarrow \mathbb{Z}$ with respect to $\hat{H}_{[0, t]}$ such that

$$
\gamma(s) \neq \gamma(s-) \Longrightarrow \mathbb{Z} \times\{0\} \leftrightarrow(\gamma(s), s-) \text { in } \hat{H}_{[0, t]}, \quad s \in[0, t] .
$$

We then let $\eta_{t}^{x}=\gamma(0)$.

It should now be clear that (2.20) indeed holds.

The following lemma describes the duality relation that exists between the ancestor process and the multitype contact process.

Lemma 2.7. (Duality) Let $\left\{\left(\eta_{t}^{x}\right)_{t \geq 0}: x \in \mathbb{Z}\right\}$ be the ancestor processes obtained from a Harris system $H$. Fix $t>0$ and let $\hat{H}_{[0, t]}$ be defined as in (2.21). Given $\xi_{0} \in\{0,1,2\}^{\mathbb{Z}}$, let $\left(\xi_{s}\right)_{0 \leq s \leq t}$ be the multitype contact process started from $\xi_{0}$ and constructed with $\hat{H}_{[0, t]}$. Then, for any $x \in \mathbb{Z}$,

$$
\begin{aligned}
& \text { if } \eta_{t}^{x}=\triangle, \text { then } \xi_{t}(x)=0 \text { and } \\
& \text { if } \eta_{t}^{x} \neq \triangle \text { and } \xi_{0}\left(\eta_{t}^{x}\right) \neq 0 \text {, then } \xi_{t}(x)=\xi_{0}\left(\eta_{t}^{x}\right) .
\end{aligned}
$$

In particular, if $\xi_{0}(x) \neq 0$ for all $x \in \mathbb{Z}$, then, with the convention that $\xi_{0}(\triangle)=0$,

$$
\left(\xi_{t}(x): x \in \mathbb{Z}\right)=\left(\xi_{0}\left(\eta_{t}^{x}\right): x \in \mathbb{Z}\right) .
$$

Proof: If $\eta_{t}^{x}=\triangle$, then $\mathbb{Z} \times\{0\} \leftrightarrow(x, t)$ in $\hat{H}_{[0, t]}$, so $\xi_{t}(x)=0$ by (2.17), proving (2.23). To prove (2.24), assume $\eta_{t}^{x}=y \in \mathbb{Z}$ and $\xi_{0}(y)=i \in\{1,2\}$. By the definition of $\eta_{t}^{x}$, there exists an infection path $\gamma$ (with respect to $\hat{H}_{[0, t]}$ ) such that (2.22) holds. Consequently,

$$
\gamma(s) \neq \gamma(s-) \Longrightarrow \xi_{s-}(\gamma(s))=0,
$$

so (2.24) follows from Lemma 2.5. The third statement is an immediate consequence of the previous two.

c.) Ancestor process started from arbitrary space-time points; concatenation property. It will be useful to consider ancestor processes started from space-time points $(x, r)$ with $r \geq 0$; we define these by

$$
\eta_{r+s}^{(x, r)}(H)=\eta_{s}^{x}(H \circ \Theta(0, r)) .
$$

When $r=0$, we will keep writing $\left(\eta_{t}^{x}\right)$ instead of $\left(\eta_{t}^{(x, 0)}\right)$. If we also have $x=0$, we simply write $\left(\eta_{t}\right)_{t \geq 0}$. Naturally, we have

$$
\eta_{t}^{(x, r)} \in\{y:(x, r) \leftrightarrow(y, t)\} \text { if } t<T^{(x, r)} \quad \text { and } \quad \eta_{t}^{(x, r)}=\triangle \text { if } t \geq T^{(x, r)} .
$$

and

$$
\left(\eta_{r+t}^{(x, r)}\right)_{t \geq 0} \stackrel{\text { (dist.) }}{=}\left(\eta_{t}^{x}\right)_{t \geq 0} \stackrel{\text { (dist.) }}{=}\left(\eta_{t}+x\right)_{t \geq 0} .
$$

The usefulness of starting the ancestor process at arbitrary space-time points is in the following result. 
Lemma 2.8. (Concatenation property). For $0 \leq r<t<t^{\prime}$, we have:

$$
\text { if } \eta_{t}^{(x, r)} \neq \triangle \text { and } \eta_{t^{\prime}}^{\left(\eta_{t}^{(x, r)}, t\right)} \neq \triangle \text {, then } \eta_{t^{\prime}}^{(x, r)}=\eta_{t^{\prime}}^{\left(\eta_{t}^{(x, r)}, t\right)} .
$$

In particular, for $r<t<\infty$,

$$
\text { if } \eta_{t}^{(x, r)} \neq \triangle \text { and } T^{\left(\eta_{t}^{(x, r)}, t\right)}=\infty \text {, then } \eta_{t^{\prime}}^{(x, r)}=\eta_{t^{\prime}}^{\left(\eta_{t}^{(x, r)}, t\right)} \text { for all } t^{\prime} \geq t .
$$

Proof: The result is an easy consequence of the following fact. Suppose $\gamma:[a, b] \rightarrow$ $\mathbb{Z}$ and $\gamma^{\prime}:[b, c] \rightarrow \mathbb{Z}$ are infection paths satisfying:

$$
\begin{aligned}
& \gamma(b)=\gamma^{\prime}(b), \\
& \gamma(s) \neq \gamma(s-) \Longrightarrow \mathbb{Z} \times\{0\} \leftrightarrow(\gamma(s), s-), \quad s \in[a, b], \\
& \gamma^{\prime}(s) \neq \gamma^{\prime}(s-) \Longrightarrow \mathbb{Z} \times\{b\} \leftrightarrow(\gamma(s), s-), \quad s \in[b, c] .
\end{aligned}
$$

Then, the concatenation $\gamma^{\prime \prime}:[a, c] \rightarrow \mathbb{Z}$, defined by

$$
\gamma^{\prime \prime}(s)= \begin{cases}\gamma(s) & \text { if } s \in[a, b], \\ \gamma^{\prime}(s) & \text { if } s \in[b, c]\end{cases}
$$

satisfies

$$
\gamma^{\prime \prime}(s) \neq \gamma^{\prime \prime}(s-) \Longrightarrow \mathbb{Z} \times\{0\} \leftrightarrow\left(\gamma^{\prime \prime}(s), s-\right), \quad s \in[a, c] .
$$

2.4. Renewal times of the ancestor process. We now recall the renewal structure from which we are able to decompose the ancestor process into pieces that are independent and identically distributed. This then allows us to find an embedded random walk in $\left(\eta_{s}\right)$ and argue that the whole of the trajectory of $\left(\eta_{s}\right)$ remains close to this embedded random walk. Most of the results of this subsection are not new (they appear in Neuhauser (1992) or Valesin (2010) or both); in an effort to balance the self-sufficiency of this paper with shortness of exposition, we will include a few key proofs and omit others.

Due to the concatenation property (2.28), it will be useful to find times $t$ for which $\left(\eta_{t}, t\right) \leftrightarrow \infty$. Let us discuss how the search for these times goes. Fix $t_{0} \geq 0$ and, on the event $\{(0,0) \leftrightarrow \infty\}$, define

$$
\begin{aligned}
& \tau=\inf \left\{t \geq t_{0}:\left(\eta_{t}, t\right) \leftrightarrow \infty\right\}, \\
& \sigma_{0}=t_{0}, \quad \sigma_{k+1}= \begin{cases}T^{\left(\eta_{\sigma_{k}}, \sigma_{k}\right)} & \text { if } \sigma_{k}<\infty \\
\infty & \text { if } \sigma_{k}=\infty\end{cases}
\end{aligned}
$$

We claim that

$$
\text { almost surely on }\{(0,0) \leftrightarrow \infty\}, \tau=\sup \left\{\sigma_{k}: \sigma_{k}<\infty\right\} \text {. }
$$

See Figure 2.2 for an illustration of the random times $\left(\sigma_{k}\right), \tau$, and of this claim. We interpret (2.31) as expressing that $\tau$ is obtained as the result of repeated attempts of having $\left(\eta_{t}, t\right) \leftrightarrow \infty$, starting at time $t_{0}$ and moving forwards in time. That is, we first check whether $\left(\eta_{t_{0}}, t_{0}\right) \leftrightarrow \infty$. If so, we have $\tau=\sigma_{0}=t_{0}$. Otherwise, we wait until time $\sigma_{1}=T^{\left(\eta_{t_{0}}, t_{0}\right)}$, that is, the first time $t$ at which the set $\left\{y:\left(\eta_{t_{0}}, t_{0}\right) \leftrightarrow\right.$ $(y, t)\}$ is empty. We then check whether or not $\left(\eta_{\sigma_{1}}, \sigma_{1}\right) \leftrightarrow \infty$; if so, we have $\tau=\sigma_{1}$, and so on. 


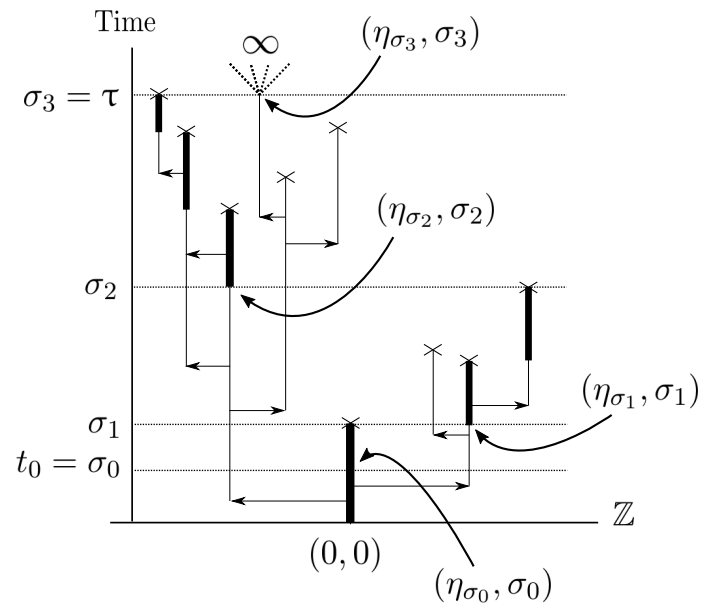

Figure 2.2. Illustration of the times $\sigma_{k}$ and $\tau$. The thick black line represents the evolution of the ancestor process. The infinity symbol on top means that $\left(\eta_{\sigma_{3}}, \sigma_{3}\right) \leftrightarrow \infty$, so that $\tau=\sigma_{3}$.

The statement (2.31) is proved as follows. If $\left(\eta_{\sigma_{k}}, \sigma_{k}\right) \leftrightarrow \infty$ for some $k$, then we clearly have $\tau \leq \sigma_{k}$. Now assume that, for some $k$ and $t$, we have $\sigma_{k} \leq t<$ $\sigma_{k+1}<\infty$. Since $t<\sigma_{k+1}=T^{\left(\eta_{\sigma_{k}}, \sigma_{k}\right)}$, we have $\left(\eta_{\sigma_{k}}, \sigma_{k}\right) \leftrightarrow \mathbb{Z} \times\{t\}$, so at time $t$, the first ancestor of $\left(\eta_{\sigma_{k}}, \sigma_{k}\right)$ is some $y \in \mathbb{Z}$ with $\left(\eta_{\sigma_{k}}, \sigma_{k}\right) \leftrightarrow(y, t)$. Hence, $T^{(y, t)} \leq T^{\left(\eta_{\sigma_{k}}, \sigma_{k}\right)}=\sigma_{k+1}<\infty$. Using the concatenation formula (2.27) with $r=0$, we also have $\eta_{t}=\eta_{t}^{\left(\eta_{\sigma_{k}}, \sigma_{k}\right)}=y$. Therefore we have proved that

$$
\sigma_{k} \leq t<\sigma_{k+1}<\infty \Longrightarrow \tau \geq t
$$

This completes the proof of (2.31).

Equation (2.27) is a key ingredient for obtaining the following result.

Lemma 2.9. There exists $c>0$ such that, for any $b>a \geq 0$, we have

$$
\mathbb{P}\left[\eta_{s} \neq \triangle \text { and }\left(\eta_{s}, s\right) \leftrightarrow \infty \text { for all } s \in[a, b]\right]<e^{-c(b-a)} .
$$

See Proposition 1 of Neuhauser (1992) (page 474) for a full proof. The idea is as follows. First note that

$$
\begin{gathered}
\mathbb{P}\left[\eta_{s} \neq \triangle \text { and }\left(\eta_{s}, s\right) \leftrightarrow \infty \text { for all } s \in[a, b],(0,0) \leftrightarrow \infty\right] \leq \mathbb{P}\left[b-a<T^{0}<\infty\right] \\
\stackrel{(2.9)}{<} e^{-c(b-a)} .
\end{gathered}
$$

Hence, it suffices to bound

$$
\mathbb{P}\left[\eta_{s} \neq \triangle \text { and }\left(\eta_{s}, s\right) \leftrightarrow \infty \text { for all } s \in[a, b],(0,0) \leftrightarrow \infty\right] .
$$

Define $\tau$ and $\left(\sigma_{k}\right)_{k=0}^{\infty}$ as in (2.29), (2.30) with $t_{0}=a$. One can then argue that $\max \left\{k: \sigma_{k}<\infty\right\}$ can be stochastically dominated by a geometric random variable with success probability $\mathbb{P}\left[T^{0}=\infty\right]$. Moreover, if $\sigma_{k}<\sigma_{k+1}<\infty$, then the law of $\sigma_{k+1}-\sigma_{k}$ has exponentially decaying tail (in other words, if $\left(\eta_{\sigma_{k}}, \sigma_{k}\right)$ does not survive to $\infty$, then it dies out quickly). Equation (2.32) is obtained by putting these ideas together. 
Given a finite set $A \subset \mathbb{Z}$, we write

$$
\tilde{\mathbb{P}}^{A}[\cdot]=\mathbb{P}[\cdot \mid(y, 0) \leftrightarrow \infty \text { for all } y \in A] .
$$

In case $A=\{x\}$, we write $\tilde{\mathbb{P}}^{x}$ instead of $\tilde{\mathbb{P}}^{\{x\}}$ and in case $x=0$, we omit the superscript. We write $\tilde{\mathbb{E}}^{A}, \tilde{\mathbb{E}}^{x}$ and $\tilde{\mathbb{E}}$ for the corresponding expectation.

Lemma 2.10. Let $t_{0}>0$ and, on the event $\{(0,0) \leftrightarrow \infty\}$, define $\tau$ as in (2.29). We then have

$$
\tilde{\mathbb{P}}[\tau<\infty]=1 .
$$

Moreover, for any $y \in \mathbb{Z}$ and events $E, F$ on Harris systems,

$\tilde{\mathbb{P}}\left[H_{[0, \tau]} \in E, \eta_{\tau}=y\right.$ and $\left.H \circ \theta(0, \tau) \in F\right]=\tilde{\mathbb{P}}\left[H_{[0, \tau]} \in E, \eta_{\tau}=y\right] \cdot \tilde{\mathbb{P}}^{y}[H \in F]$.

Proof: The first statement is a direct consequence of Lemma 2.9. For the second statement, let $\left(\sigma_{k}\right)_{k=0}^{\infty}$ be as in (2.30). Using (2.31) and the fact that $\left(\sigma_{k}\right)_{k=0}^{\infty}$ is an increasing sequence of stopping times with respect to the natural filtration of Harris systems, the left-hand side of (2.35) becomes

$$
\begin{aligned}
& \sum_{k=0}^{\infty} \tilde{\mathbb{P}}\left[\sigma_{k}<\infty ; H_{\left[0, \sigma_{k}\right]} \in E ; \eta_{\sigma_{k}}=y \text { and }\left(y, \sigma_{k}\right) \leftrightarrow \infty ; H \circ \theta\left(0, \sigma_{k}\right) \in F\right] \\
& =\mathbb{P}[(0,0) \leftrightarrow \infty]^{-1} \cdot \sum_{k=0}^{\infty} \mathbb{P}\left[\begin{array}{l}
\sigma_{k}<\infty ; H_{\left[0, \sigma_{k}\right]} \in E ; \\
\eta_{\sigma_{k}}=y \text { and }\left(y, \sigma_{k}\right) \leftrightarrow \infty ; H \circ \theta\left(0, \sigma_{k}\right) \in F
\end{array}\right] \\
& =\tilde{\mathbb{P}} y \in F] \cdot \sum_{k=0}^{\infty} \tilde{\mathbb{P}}\left[\sigma_{k}<\infty ; H_{\left[0, \sigma_{k}\right]} \in E ; \eta_{\sigma_{k}}=y \text { and }\left(y, \sigma_{k}\right) \leftrightarrow \infty\right] \\
& =\tilde{\mathbb{P}}^{y}[H \in F] \cdot \tilde{\mathbb{P}}\left[H_{[0, \tau]} \in E ; \eta_{\tau}=y\right] .
\end{aligned}
$$

Given $(z, r) \in \mathbb{Z} \times[0, \infty)$, on the event $(z, r) \leftrightarrow \infty$ we define the times

$$
\tau_{0}^{(z, r)}=r, \quad \tau_{k}^{(z, r)}=\inf \left\{t \geq \tau_{k-1}^{(z, r)}+1:\left(\eta_{t}^{(z, r)}, t\right) \leftrightarrow \infty\right\}, k \geq 1 .
$$

We write $\tau_{k}^{z}$ instead of $\tau_{k}^{(z, 0)}$ and $\tau_{k}$ instead of $\tau_{k}^{0}$. We now state three simple facts about these random times. First, it follows from (2.28) that

$$
\text { if } \tau_{1}=t \text { and } \eta_{t}=z \text {, then } \tau_{k}=\tau_{k-1}^{(z, t)} \text { for all } k \geq 1 \text {. }
$$

Second, from (2.32) it is easy to obtain

$$
\tilde{\mathbb{P}}\left[\tau_{k}<\infty \text { for all } k\right]=1 .
$$

Third, by putting (2.8) and (2.32) together, it is easy to show that

$$
\tilde{\mathbb{P}}\left[\max \left(\tau_{1}, \sup _{0 \leq s \leq \tau_{1}}\left|\eta_{s}\right|\right)>t\right] \leq e^{-c t}, \quad t>0 .
$$

Our main tool in dealing with the ancestor process is the following result.

Proposition 2.11. $\quad$ (1) Under $\tilde{\mathbb{P}}$,

$$
\left(\tau_{k+1}-\tau_{k},\left(\eta_{t}-\eta_{\tau_{k}}\right)_{\tau_{k} \leq t<\tau_{k+1}}\right), k=1,2, \ldots \text { are i.i.d. }
$$

In particular, $\left(\eta_{\tau_{k}}\right)_{k \geq 0}$ is a random walk on $\mathbb{Z}$ with increment distribution

$$
\tilde{\mathbb{P}}\left[\eta_{\tau_{k+1}}=w \mid \eta_{\tau_{k}}=z\right]=\tilde{\mathbb{P}}\left[\eta_{\tau_{1}}=w-z\right] .
$$


(2) There exist $C, c>0$ such that, for any $t \geq 0, r>0$ and $x \geq 0$,

$$
\mathbb{P}\left[\eta_{t+r} \neq \triangle, \sup _{s \in[t, t+r]}\left|\eta_{s}-\eta_{t}\right|>x\right] \leq \tilde{\mathbb{P}}\left[\max _{k \in\{1, \ldots,\lceil r\rceil\}}\left|\eta_{\tau_{k}}\right|>\frac{x}{3}\right]+C r e^{-c x}
$$

(3) Under $\tilde{\mathbb{P}}$,

$$
\frac{\eta_{t}}{\sqrt{t}} \underset{(\text { dist. })}{\stackrel{t \rightarrow \infty}{\longrightarrow}} \mathcal{N}\left(0, \sigma^{2}\right) \text { with } \sigma>0
$$

Remark 2.12. Due to (2.39), the law of $\max _{k \in\{1, \ldots, K\}}\left|\eta_{\tau_{k}}\right|$ under $\tilde{\mathbb{P}}$ is the same as that of $\max _{k \in\{1, \ldots, K\}}\left|X_{1}+\cdots+X_{k}\right|$, where $X_{1}, \ldots, X_{K}$ are independent and identically distributed random variables with law $\mathbb{P}\left[X_{i}=\cdot\right]=\tilde{\mathbb{P}}\left[\eta_{\tau_{1}}=\cdot\right]$. Due to (2.38), this increment distribution $\tilde{\mathbb{P}}\left[\eta_{\tau_{1}}=\cdot\right]$ has exponential tails. Hence, standard maximal inequalities for random walks with exponential moments are applicable; see for example Section 12.2.2 of Lawler and Limic (2010). In particular, if $\delta$ is small enough, we have $\tilde{\mathbb{E}}\left[\exp \left\{\delta\left|\eta_{\tau_{1}}\right|\right\}\right]<\infty$ and Theorem 12.2.5 in Lawler and Limic (2010) gives

$$
\tilde{\mathbb{P}}\left[\max _{k \in\{1, \ldots, K\}}\left|\eta_{\tau_{k}}\right|>x\right]<e^{-\delta x} \cdot\left(\tilde{\mathbb{E}}\left[\exp \left\{\delta\left|\eta_{\tau_{1}}\right|\right\}\right]\right)^{K}, \quad x>0
$$

Optimizing over the possible values of $\delta$ as in Corollary 12.2.7 of Lawler and Limic (2010), it can then be shown that, for some $c>0$,

$$
\left.\tilde{\mathbb{P}} \max _{k \in\{1, \ldots, K\}}\left|\eta_{\tau_{k}}\right|>x\right]<\exp \left\{-c x^{2} / K\right\}, \quad 0<x<K^{2 / 3}
$$

Proof of Proposition 2.11. A proof of part (2.39) can be found in Neuhauser (1992), but we give another one here. Let $E_{0}, \ldots, E_{k}$ be measurable subsets of $\cup_{t \geq 0} D[0, t]$, the space of finite-time trajectories that are right-continuous with left limits. We evaluate

$$
\begin{aligned}
& \tilde{\mathbb{P}}\left[\left(\eta_{s}-\eta_{\tau_{i}}\right)_{\tau_{i}<s \leq \tau_{i+1}} \in E_{i} \text { for } i=0, \ldots, k\right] \\
& =\sum_{z \in \mathbb{Z}} \tilde{\mathbb{P}}\left[\left(\eta_{s}\right)_{0 \leq s \leq \tau_{1}} \in E_{0}, \eta_{\tau_{1}}=z,\left(\eta_{s}-\eta_{\tau_{i}}\right)_{\tau_{i}<s \leq \tau_{i+1}} \in E_{i} \text { for } i=1, \ldots, k\right] .
\end{aligned}
$$

Now, by (2.28) and (2.36),

on $\left\{(0,0) \leftrightarrow \infty, \eta_{\tau_{1}}=z\right\}$

we have $\eta_{s}=\eta_{s}^{\left(z, \tau_{1}\right)}$ for all $s \geq \tau_{1}$ and $\tau_{k}=\tau_{k-1}^{\left(z, \tau_{1}\right)}$ for all $k \geq 1$. 
Applying these identities and Lemma 2.10, we obtain that (2.44) is equal to

$$
\begin{aligned}
& \sum_{z \in \mathbb{Z}} \tilde{\mathbb{P}}\left.\left(\eta_{s}\right)_{0 \leq s \leq \tau_{1}} \in E_{0}, \eta_{\tau_{1}}=z\right] \\
& \times \tilde{\mathbb{P}}^{z}\left[\left(\eta_{s}^{z}-\eta_{\tau_{i}}^{z}\right)_{\tau_{i}<s \leq \tau_{i+1}} \in E_{i+1} \text { for } i=0, \ldots, k-1\right] \\
&=\sum_{z \in \mathbb{Z}} \tilde{\mathbb{P}} {\left[\left(\eta_{s}\right)_{0 \leq s \leq \tau_{1}} \in E_{0}, \eta_{\tau_{1}}=z\right] } \\
& \quad \times \tilde{\mathbb{P}}\left[\left(\eta_{s}-\eta_{\tau_{i}}\right)_{\tau_{i}<s \leq \tau_{i+1}} \in E_{i+1} \text { for } i=0, \ldots, k-1\right] \\
&=\tilde{\mathbb{P}}\left[\left(\eta_{s}\right)_{0 \leq s \leq \tau_{1}} \in E_{0}\right] \cdot \tilde{\mathbb{P}}\left[\left(\eta_{s}-\eta_{\tau_{i}}\right)_{\tau_{i}<s \leq \tau_{i+1}} \in E_{i+1} \text { for } i=0, \ldots, k-1\right] .
\end{aligned}
$$

We now iterate this computation to obtain (2.39).

For the remaining statements, we will need a definition. On the event $\{(0,0) \leftrightarrow$ $\infty\}$, let

$$
N_{t}=\max \left\{k: \tau_{k} \leq t\right\}, \quad t \geq 0 .
$$

Since we have $\tau_{k+1}-\tau_{k} \geq 1$ for all $k$, we obtain

$$
N_{t+r}-N_{t} \leq\lceil r\rceil \text { for any } t \geq 0, r>0 .
$$

We now turn to (2.40). Recalling that under $\tilde{\mathbb{P}}$, we almost surely have $(0,0) \leftrightarrow \infty$, the left-hand side of (2.40) is less than

$$
\mathbb{P}\left[t+r<T^{0}<\infty, \sup _{s \in[t, t+r]}\left|\eta_{s}-\eta_{t}\right|>x\right]+\tilde{\mathbb{P}}\left[\sup _{s \in[t, t+r]}\left|\eta_{s}-\eta_{t}\right|>x\right] .
$$

The first term in (2.46) is less than

$$
\mathbb{P}\left[T^{0}<\infty, \sup _{0 \leq s \leq T^{0}}\left|\eta_{s}\right|>\frac{x}{2}\right] \leq \mathbb{P}\left[\frac{x}{2 \kappa}<T^{0}<\infty\right]+\mathbb{P}\left[\sup _{s \in[0, x /(2 \kappa)]}\left|\eta_{s}\right|>\frac{x}{2}\right],
$$

where $\kappa$ is as in (2.8). Then, (2.8) and (2.9) show that the sum is less than $e^{-c x}$ for some $c>0$. Noting that $[t, t+r] \subset\left[\tau_{N_{t}}, \tau_{N_{t}+\lceil r\rceil+1}\right]$, the second term in (2.46) is less than

$$
\begin{aligned}
\tilde{\mathbb{P}}\left[\sup _{s \in\left[\tau_{N_{t}}, \tau_{N_{t}+1}\right]}\left|\eta_{s}-\eta_{\tau_{N_{t}}}\right|>\frac{x}{3}\right] \\
\quad+\tilde{\mathbb{P}}\left[\max _{k \in\left\{N_{t}+2, \ldots, N_{t}+\lceil r\rceil+1\right\}}\left|\eta_{\tau_{k}}-\eta_{\tau_{N_{t}+1}}\right|>\frac{x}{3}\right] \\
\quad+\tilde{\mathbb{P}}\left[\max _{k \in\left\{N_{t}+1, \ldots, N_{t}+\lceil r\rceil+1\right\}} \sup _{s \in\left[\tau_{k}, \tau_{k+1}\right]}\left|\eta_{s}-\eta_{\tau_{k}}\right|>\frac{x}{3}\right] .
\end{aligned}
$$

A computation carried out in Lemma 2.5 of Valesin (2010) shows that the first term of (2.47) is bounded above by $e^{-c x}$ for some $c>0$ (see equation (2.10) in that paper). Next, Lemma 2.10 implies that the second term in (2.47) is equal to

$$
\tilde{\mathbb{P}}\left[\max _{k \in\{1, \ldots,\lceil r\rceil\}}\left|\eta_{\tau_{k}}\right|>\frac{x}{3}\right] .
$$


Finally, again by Lemma 2.10, the third term in (2.47) is equal to

$$
\begin{aligned}
& \left.\tilde{\mathbb{P}} \max _{k \in\{1, \ldots,\lceil r\rceil+1\}} \sup _{s \in\left[\tau_{k}, \tau_{k+1}\right]}\left|\eta_{s}-\eta_{\tau_{k}}\right|>\frac{x}{3}\right] \\
& \stackrel{(2.39)}{\leq}(\lceil r\rceil+1) \cdot \tilde{\mathbb{P}}\left[\sup _{s \in\left[0, \tau_{1}\right]}\left|\eta_{s}\right|>\frac{x}{3}\right] \stackrel{(2.38)}{\leq}(\lceil r\rceil+1) \cdot e^{-c x} .
\end{aligned}
$$

Finally, let us prove (2.41). Denote

$$
\mu=\left(\tilde{\mathbb{E}} \tau_{1}\right)^{-1} \stackrel{(2.38)}{<} \infty
$$

In Lemma 2.5 in Valesin (2010), it is shown that

$$
\tilde{\mathbb{P}}\left[\sup \left\{\left|\eta_{s}-\eta_{\tau_{N_{t}}}\right|: \tau_{N_{t}} \leq s \leq \tau_{N_{1}+1}\right\}>x\right] \leq e^{-c x}, \quad t>0, x>0 .
$$

We write

$$
\frac{\eta_{t}}{\sqrt{t}}=\frac{\eta_{\lfloor t / \mu\rfloor}}{\sqrt{t}}+\frac{\eta_{N_{t}}-\eta_{\lfloor t / \mu\rfloor}}{\sqrt{t}}+\frac{\eta_{t}-\eta_{N_{t}}}{\sqrt{t}}
$$

By (2.39) and the Central Limit Theorem, $\frac{\eta_{\lfloor t / \mu\rfloor}}{\sqrt{t}}$ converges in distribution, as $t \rightarrow \infty$, to $\mathcal{N}\left(0, \sigma^{2}\right)$ with $\sigma>0$. Using (2.48), we have that $\frac{\eta_{t}-\eta_{N_{t}}}{\sqrt{t}}$ converges in probability, as $t \rightarrow \infty$, to zero. Hence, (2.41) will follow if we prove that the remaining term also satisfies

$$
\frac{\eta_{N_{t}}-\eta_{\lfloor t / \mu\rfloor}}{\sqrt{t}} \underset{(\text { prob. })}{\stackrel{t \rightarrow \infty}{\longrightarrow}} 0
$$

With this aim, fix $\varepsilon>0$. For any $\delta>0$ we have

$$
\begin{aligned}
\tilde{\mathbb{P}}\left[\frac{\eta_{N_{t}}-\eta_{\lfloor t / \mu\rfloor}}{\sqrt{t}}>\varepsilon\right] & \leq \tilde{\mathbb{P}}\left[\frac{N_{t}}{t}-\frac{1}{\mu}>\delta\right] \\
& +\tilde{\mathbb{P}}\left[\frac{\eta_{N_{t}}-\eta_{\lfloor t / \mu\rfloor}}{\sqrt{t}}>\varepsilon, N_{t} \in\left[\frac{t}{\mu}-\delta t, \frac{t}{\mu}\right]\right] \\
& +\tilde{\mathbb{P}}\left[\frac{\eta_{N_{t}}-\eta_{\lfloor t / \mu\rfloor}}{\sqrt{t}}>\varepsilon, N_{t} \in\left[\frac{t}{\mu}, \frac{t}{\mu}+\delta t\right]\right] .
\end{aligned}
$$

By the Renewal Theorem, $\tilde{\mathbb{P}}\left[\frac{N_{t}}{t}-\frac{1}{\mu}>\delta\right] \rightarrow 0$ as $t \rightarrow \infty$. Next,

$$
\begin{aligned}
\tilde{\mathbb{P}}\left[\frac{\left|\eta_{N_{t}}-\eta_{\lfloor t / \mu\rfloor}\right|}{\sqrt{t}}>\varepsilon, N_{t} \in\left[\frac{t}{\mu}, \frac{t}{\mu}+\delta t\right]\right] & \leq \tilde{\mathbb{P}}\left[\max _{\frac{t}{\mu} \leq i \leq \frac{t}{\mu}+\delta t}\left|\eta_{\tau_{i}}-\eta_{\tau_{\lfloor t / \mu\rfloor}}\right|>\varepsilon \sqrt{t}\right] \\
& \leq \delta t \frac{\operatorname{Var}\left(\eta_{\tau_{1}}\right)}{\varepsilon^{2} t}=\delta \frac{\operatorname{Var}\left(\eta_{\tau_{1}}\right)}{\varepsilon^{2}},
\end{aligned}
$$

where the last inequality is an application of Kolmogorov's Inequality. The above can be made arbitrarily small by taking $\delta$ small (depending on $\varepsilon$ ). The other term in (2.50) is then treated similarly, and the proof of (2.49) is now complete.

In Valesin (2010), results are obtained about the joint behavior of two or more ancestor processes. The method used to obtain such results involved studying renewal times that are more complicated than the $\tau_{k}^{x}$ defined above. We will not present the details here. Rather, let us just mention that, while a single ancestor behaves closely to a random walk (as outlined above), a larger amount of ancestors, when considered jointly, behave closely to a system of coalescing random walks (that 
is, a system of random walkers that move independently with the added rule that two walkers that occupy the same position merge into a single walker). Taking advantage of this comparison, one can then obtain for ancestor processes several estimates that hold for coalescing random walks. In particular, in Lemma 3.2 in Valesin (2010), it is shown that

$$
\begin{aligned}
& \text { there exists } C>0 \text { such that } \\
& \mathbb{P}\left[\eta_{t}^{x}, \eta_{t}^{y} \neq \triangle, \eta_{t}^{x} \neq \eta_{t}^{y}\right] \leq \frac{C|x-y|}{\sqrt{t}}, x, y \in \mathbb{Z}, t>0 .
\end{aligned}
$$

Using this result, it is then possible to show that the density of the set of all ancestors at time $t,\left\{\eta_{t}^{x}: x \in \mathbb{Z}\right\} \cap \mathbb{Z}$, goes to zero as $t \rightarrow \infty$ (see Proposition 3.5 in Valesin, 2010), so that

$$
\text { for all finite } I \subset \mathbb{Z}, \mathbb{P}\left[\left\{\eta_{t}^{x}: x \in \mathbb{Z}\right\} \cap I \neq \varnothing\right] \stackrel{t \rightarrow \infty}{\longrightarrow} 0 .
$$

Finally, we will need the bound

$$
\begin{aligned}
& \text { for any } u>0 \text { there exists } C>0 \text { such that, } \\
& \text { for } t \text { large enough and any } x<y, \\
& \mathbb{P}\left[\eta_{s}^{x}, \eta_{s}^{y} \neq \triangle, \eta_{s}^{x}>\eta_{s}^{y}+u \sqrt{t} \text { for some } s \leq t\right]<\frac{C}{\sqrt{t}} .
\end{aligned}
$$

For coalescing random walks having symmetric jump distribution with finite third moments, this estimate is given by Lemma 2.0.4 in Sun (2005). As $\left(\eta_{t}^{x}\right)$ and $\left(\eta_{t}^{y}\right)$ are not exactly coalescing random walks, the proof of the mentioned lemma has to be adapted to the present context. Given the method of proof of Theorem 6.1 in Valesin (2010), this adaption does not involve anything new, so we do not include it here.

2.5. Interface. Given $\xi \in\{0,1,2\}^{\mathbb{Z}}$, we write

$$
r(\xi)=\sup \{x \in \mathbb{Z}: \xi(x)=1\}, \quad \ell(\xi)=\inf \{x \in \mathbb{Z}: \xi(x)=2\} .
$$

Define

$$
\Omega=\left\{\begin{aligned}
\xi \in\{0,1,2\}^{\mathbb{Z}}: & \#\{x<0: \xi(x)=1\}=\#\{x>0: \xi(x)=2\}=\infty, \\
& \#\{x<0: \xi(x)=2\}<\infty, \#\{x>0: \xi(x)=1\}<\infty
\end{aligned}\right\} ;
$$

in particular, $r(\xi)<\infty$ and $\ell(\xi)>-\infty$ for any $\xi \in \Omega$.

As mentioned in the Introduction, $\left(\xi_{t}^{h}\right)_{t \geq 0}$ denotes the contact process started from the heaviside configuration, (1.5), and

$$
r_{t}=r\left(\xi_{t}^{h}\right), \quad \ell_{t}=\ell\left(\xi_{t}^{h}\right), \quad i_{t}=\left(r_{t}+\ell_{t}\right) / 2, \quad t \geq 0 .
$$

The interval delimited by $r_{t}$ and $\ell_{t}$ is the interface, and $i_{t}$ is the interface position, at time $t$. Using (2.8), it is easy to show that, almost surely,

$$
\xi_{t}^{h} \in \Omega \text { for all } t \geq 0 .
$$

It will be useful to have the following rough bound on the displacement of $r_{t}$ and $\ell_{t}$.

Lemma 2.13. For any $\varepsilon>0$ and $\sigma>0$ there exists $S_{0}>0$ such that, if $S \geq S_{0}$ and $\xi_{0}$ satisfies $\xi_{0} \equiv 2$ on $(0, \infty)$, then with probability larger than $1-\varepsilon$,

$$
\text { for any } t \geq 0, r\left(\xi_{t}\right), \ell\left(\xi_{t}\right) \leq S+\sigma t \text {. }
$$


Proof: It is sufficient to prove the result for $\sigma \in(0, \bar{\beta})$, where $\bar{\beta}$ is the constant that appears in Lemmas 2.1 and 2.3. We fix $\sigma^{\prime}, \sigma^{\prime \prime}$ with

$$
0<\sigma^{\prime}<\sigma^{\prime \prime}<\sigma \text {. }
$$

Using the joint construction of the multitype contact process and the ancestor processes (as described in Subsection 2.3 and in particular equation (2.25)) together with the assumption that $\xi_{0} \equiv 2$ on $(0, \infty)$ and Claim 2.6, we have

$$
\mathbb{P}\left[\xi_{t}(x)=1\right] \leq \mathbb{P}\left[\xi_{t}^{h}(x)=1\right]=\mathbb{P}\left[\eta_{t}^{x} \neq \triangle, \eta_{t}^{x} \leq 0\right] .
$$

If $x \geq 0$, the right-hand side is smaller than or equal to

$$
\begin{aligned}
& \mathbb{P}\left[\eta_{t}^{x} \neq \Delta,\left|\eta_{t}^{x}-x\right| \geq x\right] \\
& =\mathbb{P}\left[\eta_{t} \neq \Delta,\left|\eta_{t}\right| \geq x\right] \stackrel{(2.40)}{\leq} \tilde{\mathbb{P}}\left[\max _{k \in\{1, \ldots,[t]\}}\left|\eta_{\tau_{k}}\right|>\frac{x}{3}\right]+C t e^{-c x} .
\end{aligned}
$$

Combining the previous two inequalities with a union bound (see also Remark 2.12), we get

$$
\begin{aligned}
& \mathbb{P}\left[r\left(\xi_{t}\right) \geq \frac{S}{3}+\sigma^{\prime} t \text { for some } t \in \mathbb{Z}_{+}\right] \\
& =\mathbb{P}\left[\xi_{t}(x)=1 \text { for some } t \in \mathbb{Z}_{+} \text {and } x \geq \frac{S}{3}+\sigma^{\prime} t\right]<\frac{\varepsilon}{3}
\end{aligned}
$$

if $S$ is large enough. We then bound

$$
\begin{aligned}
& \mathbb{P}\left[r\left(\xi_{t}\right)<S / 3+\sigma^{\prime} t \text { and } r\left(\xi_{s}\right) \geq 2 S / 3+\sigma^{\prime \prime} t \text { for some } s \in[t, t+1]\right] \\
& \leq \mathbb{P}\left[\left(-\infty, S / 3+\sigma^{\prime} t\right) \times\{t\} \leftrightarrow\left[2 S / 3+\sigma^{\prime \prime} t, \infty\right) \times[t, t+1]\right]<e^{-c(S+\sigma t / 2)}
\end{aligned}
$$

for some $c>0$, by a comparison with a Poisson random variable (describing the number of arrivals in a certain space-time region; we omit the details). Together with (2.55), this shows that, if $S$ is large enough,

$$
\mathbb{P}\left[r\left(\xi_{t}\right)>\frac{2 S}{3}+\sigma^{\prime \prime} t \text { for some } t \geq 0\right]<\varepsilon / 2 .
$$

By Lemma 2.3 and (2.16), increasing $S$ if necessary we have

$$
\mathbb{P}\left[\xi_{t} \equiv 0 \text { on }\left[\frac{2 S}{3}+\sigma^{\prime \prime} t, S+\sigma t\right] \text { for some } t \geq 0\right]<\varepsilon / 2 .
$$

To conclude, using (2.56) and (2.57),

$$
\begin{aligned}
& \mathbb{P}\left[r\left(\xi_{t}\right), \ell\left(\xi_{t}\right) \leq S+\sigma t \text { for all } t \geq 0\right] \\
& \geq \mathbb{P}\left[\begin{array}{c}
\text { for all } t \geq 0, r\left(\xi_{t}\right)<\frac{2 S}{3}+\sigma^{\prime \prime} t \\
\text { and } \xi_{t}(x) \neq 0 \text { for some } x \in\left[\frac{2 S}{3}+\sigma^{\prime \prime} t, S+\sigma t\right]
\end{array}\right]>1-\varepsilon .
\end{aligned}
$$

Given a Harris system $H$ and $s \geq 0$, we define the regenerated interface process $\left(i_{t}^{s}\right)_{t \geq s}$ as follows:

for any $x \in \mathbb{Z}$ and $t \geq s$, on $\left\{\left\lfloor i_{s}(H)\right\rfloor=x\right\}$, let $i_{t}^{s}(H)=x+i_{t-s}(H \circ \theta(x, s))$.

Let us explain this definition in words. Using the Harris system $H$, we construct the multitype contact process started from the heaviside configuration $\xi_{0}^{h}$ and evolve it 
up to time $s$, obtaining the configuration $\xi_{s}^{h}$ with corresponding interface position $i_{s}$. Then, we artificially put 1 's on $\left(-\infty,\left\lfloor i_{s}\right\rfloor\right]$ and 2 's on $\left(\left\lfloor i_{s}\right\rfloor, \infty\right)$ and, using $H_{[s, t]}$, we continue evolving the process; the resulting interface position at time $t$ is $i_{t}^{s}$. Note in particular that

$$
\left(i_{s+t}^{s}-i_{s}^{s}\right)_{t \geq 0} \stackrel{(\text { dist. })}{=}\left(i_{t}\right)_{t \geq 0} \quad \text { and } \quad\left|i_{s}^{s}-i_{s}\right|=0 \text { or } \frac{1}{2} ;
$$

the first equality follows from translation invariance of $H$ in space and time. In Section 5, we will prove:

Theorem 2.14. For any $\varepsilon>0$ there exists $K>0$ such that, for any $s \geq 0$,

$$
\mathbb{P}\left[\left|i_{t}^{s}-i_{t}\right|<K \text { for all } t \geq s\right]>1-\varepsilon .
$$

As a consequence we obtain

Corollary 2.15. For any $\varepsilon>0$ and $r>0$ there exists $K>0$ such that

$$
\mathbb{P}\left[\sup _{s \leq t \leq s+r}\left|i_{t}-i_{s}\right|>K\right]<\varepsilon \quad \text { for all } s \geq 0 .
$$

Proof: For any $s, r, K$, by (2.59),

$$
\begin{aligned}
& \mathbb{P}\left[\sup _{t \in[s, s+r]}\left|i_{t}-i_{s}\right|>K\right] \\
& \leq \mathbb{P}\left[\sup _{t \in[s, \infty)}\left|i_{t}^{s}-i_{t}\right|>K / 2\right]+\mathbb{P}\left[\sup _{t \in[0, r]}\left|i_{t}\right|>(K-1) / 2\right] .
\end{aligned}
$$

Now, for fixed $r$, the second term vanishes as $K \rightarrow \infty$, and the first term does so as well by Theorem 2.14.

\section{Convergence of finite-dimensional projections}

The goal of this section is to prove Proposition 1.3. Using Theorem 2.14, we will be able to deduce (at the end of this section) convergence of finite-dimensional projections from convergence of one-dimensional projections. Convergence of onedimensional projections is the content of Proposition 3.3 below. It will depend on two preliminary lemmas. Lemma 3.1 is quite simple and states that the probability that the interface is located at any (deterministic) position $x \in \frac{1}{2} \mathbb{Z}$ tends to 0 as $t \rightarrow \infty$ (uniformly on $x$ ). Lemma 3.2 is much more involved and makes sense of the following heuristic reasoning. Suppose that, for some $x \in \frac{1}{2} \mathbb{Z}$ and some large $t>0$, we observe $\xi_{t}^{h}(x)=1$. Then, there are only two possibilities: either $x$ is located at the left of the interface (that is, $x<\min \left\{r_{t}, \ell_{t}\right\}$ ) or $x$ is located inside the interface (that is, $\min \left\{r_{t}, \ell_{t}\right\} \leq x \leq \max \left\{r_{t}, \ell_{t}\right\}$ ). Using Lemma 3.1 and tightness of the interface size (Theorem 1.1), we will argue that the second alternative has negligible probability as $t \rightarrow \infty$. This will allow us to approximate the probability of $\left\{i_{t}>x \sqrt{t}\right\}$ from knowledge of the distribution of $\xi_{t}^{h}(\lfloor x \sqrt{t}\rfloor)$.

Lemma 3.1. For any $\varepsilon>0$ there exists $t_{0}>0$ such that

$$
\mathbb{P}\left[i_{t}=x\right]<\varepsilon \text { for any } x \in \frac{1}{2} \mathbb{Z}, t \geq t_{0} .
$$


Proof: Let $\varepsilon>0$. By (1.7), we can obtain $L>0$ such that for all $t, \mathbb{P}\left[\left|r_{t}-\ell_{t}\right|>\right.$ $L]<\varepsilon / 2$. For any $t$ and $x$ we have

$$
\begin{aligned}
\mathbb{P}\left[i_{t}=x\right] \leq & \mathbb{P}\left[\left|r_{t}-\ell_{t}\right|>L\right] \\
& +\mathbb{P}\left[\text { there exist } z, w \in[x-L, x+L]: \xi_{t}^{h}(z)=1, \xi_{t}^{h}(w)=2\right] .
\end{aligned}
$$

Switching to the dual process, the second probability can be written as

$\mathbb{P}$ [there exist $\left.z, w \in[x-L, x+L]: \eta_{t}^{z}, \eta_{t}^{w} \neq \triangle, \eta_{t}^{z} \leq 0, \eta_{t}^{w}>0\right]$

$$
\leq \sum_{z, w \in[x-L, x+L]} \mathbb{P}\left[\eta_{t}^{z}, \eta_{t}^{w} \neq \triangle, \eta_{t}^{z} \neq \eta_{t}^{w}\right] .
$$

By (2.51), when $t$ is large enough the sum is smaller than $\varepsilon / 2$ for any $x$, so we are done.

Lemma 3.2. For any $\varepsilon>0$ there exists $t_{0}>0$ such that

$$
\left|\mathbb{P}\left[i_{t}>x \sqrt{t}\right]-\mathbb{P}\left[\xi_{t}^{h}(\lfloor x \sqrt{t}\rfloor)=1 \mid \xi_{t}^{h}(\lfloor x \sqrt{t}\rfloor) \neq 0\right]\right|<\varepsilon \text { for any } x \in \mathbb{R} \text { and } t \geq t_{0} .
$$

Proof: Fix $\varepsilon>0$. Using (2.9), we can choose $S>0$ such that

$$
\mathbb{P}\left[S<T^{0}<\infty\right]<\varepsilon
$$

Using Corollary 2.15, we then choose $S^{\prime}>0$ such that

$$
\mathbb{P}\left[\text { there exists } s \in[t, t+S]:\left|i_{s}-i_{t}\right| \geq S^{\prime}\right]<\varepsilon \text { for all } t \geq 0 .
$$

Increasing $S^{\prime}$ if necessary, by (1.7) we can also assume that

$$
\mathbb{P}\left[\left|r_{t}-\ell_{t}\right|>S^{\prime}\right]<\varepsilon \text { for all } t \geq 0 ;
$$

Finally, using Lemma 3.1, we can choose $t_{0}>S$ such that

$$
\mathbb{P}\left[i_{t} \in\left[x-S^{\prime}, x+S^{\prime}\right]\right]<\varepsilon \quad \text { for all } t \geq 0 \text { and } x \in \mathbb{Z} .
$$

Now fix $t \geq t_{0}$ and $x \in \mathbb{R}$. Denoting by $\triangle$ the symmetric difference between sets, we have the following estimates:

$$
\begin{gathered}
\mathbb{P}\left[\left\{\xi_{t}^{h}(\lfloor x \sqrt{t}\rfloor)=1\right\} \triangle\left\{\xi_{t}^{h}(\lfloor x \sqrt{t}\rfloor) \neq 0, i_{t}>x \sqrt{t}\right\}\right] \\
\leq \mathbb{P}\left[\left|i_{t}-x \sqrt{t}\right| \leq S^{\prime}\right]+\mathbb{P}\left[\left|r_{t}-\ell_{t}\right|>S^{\prime}\right] \stackrel{(3.5),(3.6)}{\leq} 2 \varepsilon ; \\
\mathbb{P}\left[\left\{i_{t-S}>x \sqrt{t}\right\} \Delta\left\{i_{t}>x \sqrt{t}\right\}\right] \\
\leq \mathbb{P}\left[\left|i_{t}-x \sqrt{t}\right| \leq S^{\prime}\right]+\mathbb{P}\left[\left|i_{t-S}-x \sqrt{t}\right| \leq S^{\prime}\right]+\mathbb{P}\left[\left|i_{t}-i_{t-S}\right|>S^{\prime}\right] \\
\mathbb{P}[\mathbb{Z} \times\{t-S\} \leftrightarrow(x \sqrt{t}, t), \mathbb{Z} \times\{0\} \leftrightarrow(x \sqrt{t}, t)] \\
\leq \mathbb{P}[(0,0) \leftrightarrow \mathbb{Z} \times\{S\},(0,0) \leftrightarrow \leftrightarrow \infty \stackrel{(3.6)}{<} 3 \varepsilon ;
\end{gathered}
$$

the first inequality in (3.9) follows from duality (see equation (2.7)) and translation invariance of the Harris system in space and time. With these bounds at hand, we are ready to prove the statement of the lemma. In the following computation, 
the symbol $\approx$ means that the absolute value of the difference between the left-hand side and the right-hand side is at most $5 \varepsilon$.

$$
\begin{aligned}
\mathbb{P}\left[\xi_{t}^{h}(\lfloor x \sqrt{t}\rfloor)=1\right] & \stackrel{(3.7)}{\approx} \mathbb{P}\left[\xi_{t}^{h}(\lfloor x \sqrt{t}\rfloor) \neq 0, i_{t}>x \sqrt{t}\right] \\
& \stackrel{(3.8),(3.9)}{\approx} \mathbb{P}\left[\mathbb{Z} \times\{t-S\} \leftrightarrow(\lfloor x \sqrt{t}\rfloor, t), i_{t-S}>x \sqrt{t}\right] \\
& =\mathbb{P}[\mathbb{Z} \times\{t-S\} \leftrightarrow(\lfloor x \sqrt{t}\rfloor, t)] \cdot \mathbb{P}\left[i_{t-S}>x \sqrt{t}\right] \\
& \stackrel{(3.9)}{\approx} \mathbb{P}\left[\xi_{t}^{h}(\lfloor x \sqrt{t}\rfloor) \neq 0\right] \cdot \mathbb{P}\left[i_{t}>x \sqrt{t}\right] .
\end{aligned}
$$

We then have

$$
\begin{aligned}
& \left|\mathbb{P}\left[i_{t}>x \sqrt{t}\right]-\mathbb{P}\left[\xi_{t}^{h}(\lfloor x \sqrt{t}\rfloor)=1 \mid \xi_{t}^{h}(\lfloor x \sqrt{t}\rfloor) \neq 0\right]\right| \\
& =\mathbb{P}\left[\xi_{t}^{h}(\lfloor x \sqrt{t}\rfloor) \neq 0\right]^{-1} \cdot\left|\mathbb{P}\left[\xi_{t}^{h}(\lfloor x \sqrt{t}\rfloor) \neq 0\right] \cdot \mathbb{P}\left[i_{t}>x \sqrt{t}\right]-\mathbb{P}\left[\xi_{t}^{h}(\lfloor x \sqrt{t}\rfloor)=1\right]\right| \\
& \leq \mathbb{P}\left[T^{0}=\infty\right]^{-1} \cdot 15 \varepsilon,
\end{aligned}
$$

that is, at most a universal constant times $\varepsilon$. This completes the proof.

Proposition 3.3. As $t \rightarrow \infty, \frac{i_{t}}{\sqrt{t}}$ converges in distribution to $\mathcal{N}\left(0, \sigma^{2}\right)$, where $\sigma$ is as in (2.41).

Proof: The statement follows from (2.41), Lemma 3.2 and the fact that

$$
\mathbb{P}\left[\xi_{t}^{h}(\lfloor x \sqrt{t}\rfloor)=1 \mid \xi_{t}^{h}(\lfloor x \sqrt{t}\rfloor) \neq 0\right]=\tilde{\mathbb{P}}^{\lfloor x \sqrt{t}\rfloor}\left[\eta_{t}^{\lfloor x \sqrt{t}\rfloor} \leq 0\right]=\tilde{\mathbb{P}}^{0}\left[\frac{\eta_{t}^{0}}{\sqrt{t}} \leq-\frac{\lfloor x \sqrt{t}\rfloor}{\sqrt{t}}\right] .
$$

Proof of Proposition 1.3. Fix $0<a_{1}<\ldots<a_{k}$. We have

$$
\begin{aligned}
& t^{-1 / 2}\left(i_{a_{1} t}, i_{a_{2} t}-i_{a_{1} t}, \ldots, i_{a_{k} t}-i_{a_{k-1} t}\right) \\
& =t^{-1 / 2}\left(i_{a_{1} t}, i_{a_{2} t}^{a_{1} t}-i_{a_{1} t}^{a_{1} t}, \ldots, i_{a_{k} t}^{a_{k-1} t}-i_{a_{k-1} t}^{a_{k-1} t}\right) \\
& +t^{-1 / 2}\left(0, i_{a_{2} t}-i_{a_{2} t}^{a_{1} t}+i_{a_{1} t}^{a_{1} t}-i_{a_{1} t}, \ldots, i_{a_{k} t}-i_{a_{k} t}^{a_{k-1} t}+i_{a_{k-1} t}^{a_{k-1} t}-i_{a_{k-1} t}\right) .
\end{aligned}
$$

Theorem 2.14 implies that the term in (3.11) converges to zero in probability as $t \rightarrow \infty$. Since the elements of the vector in (3.10) are independent and satisfy

$$
i_{a_{i+1} t}^{a_{i} t}-i_{a_{i} t}^{a_{i} t} \stackrel{\text { (dist.) }}{=} i_{\left(a_{i+1}-a_{i}\right) t}
$$

(by (2.59)), Proposition 3.3 shows that (3.10) converges in distribution, as $t \rightarrow \infty$, to the distribution prescribed in Proposition 1.3. 


\section{Tightness in $D$}

Most of the effort in this section will go into proving the following uniform bound on the displacement of the interface position.

Lemma 4.1. For any $\varepsilon>0$ there exists $U>0$ such that, for large enough $t$ and any $r>0$,

$$
\mathbb{P}\left[\sup _{r \leq s \leq r+t}\left|i_{s}-i_{r}\right| \leq U \sqrt{t}\right]>1-\frac{\varepsilon}{U^{2}} .
$$

The proof will depend on several preliminary results. Before turning to them, let us first explain how Lemma 4.1 allows us to conclude.

Proof of Proposition 1.4. For each $t>0$, define the process $X^{(t)}$ by

$$
X_{s}^{(t)}=t^{-1 / 2} \cdot i_{s t}, \quad s \geq 0 .
$$

We want to show that the family of processes $\left\{X^{(t)}: t \geq 0\right\}$ is tight in $D[0, \infty)$. As explained in Section 16 of Billingsley (1999), it is sufficient to prove that

for all $m>0$ and $\varepsilon>0$ there exists $\delta>0$ and $t_{0}>0$ such that

$$
\mathbb{P}\left[\sup _{k \in\{0, \ldots,\lfloor m / \delta\rfloor\}} \sup _{s \in[k \delta,(k+1) \delta]}\left|X_{s}^{(t)}-X_{k \delta}^{(t)}\right|>\varepsilon\right]<\varepsilon \text { for all } t \geq t_{0} .
$$

By the identity $X_{m}^{(t)}=\sqrt{m} \cdot X_{1}^{(m t)}$, it is sufficient to treat $m=1$. Then the above condition becomes

for all $\varepsilon>0$ there exists $\delta>0$ such that, for large enough $t$,

$$
\mathbb{P}\left[\sup _{k \in\{0, \ldots,\lfloor 1 / \delta\rfloor\}} \sup _{s \in[k \delta t,(k+1) \delta t]}\left|i_{s}-i_{k \delta t}\right|>\varepsilon \sqrt{t}\right]<\varepsilon .
$$

Given $\varepsilon>0$, using Lemma 4.1, we can find $U>0$ and $t_{0}>0$ such that, if $t \geq t_{0}$,

$$
\sup _{r \geq 0} \mathbb{P}\left[\sup _{r \leq s \leq r+t}\left|i_{s}-i_{r}\right|>U \sqrt{t}\right]<\frac{\varepsilon^{3}}{U^{2}} .
$$

Now, set $\delta=(\varepsilon / U)^{2}$. We then have, for $t \geq t_{0} / \delta$ and $t^{\prime}=\delta t$,

$$
\begin{aligned}
& \mathbb{P}\left[\sup _{k \in\{0, \ldots,\lfloor 1 / \delta\rfloor\}} \sup _{s \in[k \delta t,(k+1) \delta t]}\left|i_{s}-i_{k \delta t}\right|>\varepsilon \sqrt{t}\right] \\
& \leq \frac{1}{\delta} \cdot \sup _{r \geq 0} \mathbb{P}\left[\sup _{s \in[r, r+\delta t]}\left|i_{s}-i_{r}\right|>\varepsilon \sqrt{t}\right] \\
& =\frac{1}{\delta} \cdot \sup _{r \geq 0} \mathbb{P}\left[\sup _{s \in\left[r, r+t^{\prime}\right]}\left|i_{s}-i_{r}\right|>\varepsilon \sqrt{\frac{t^{\prime}}{\delta}}\right] \\
& =\frac{U^{2}}{\varepsilon^{2}} \cdot \sup _{r \geq 0} \mathbb{P}\left[\sup _{s \in\left[r, r+t^{\prime}\right]}\left|i_{s}-i_{r}\right|>U \sqrt{t^{\prime}}\right] \stackrel{(4.3)}{<} \varepsilon
\end{aligned}
$$

as required in (4.2).

Our first step towards the proof of Lemma 4.1 are the following generalizations of Lemmas 2.9 and 2.10. Since the proofs are line-by-line repetitions of the proofs of these earlier results, we omit them. 
Lemma 4.2. (1) There exists $c>0$ such that the following holds. For any $\left(x_{1}, r_{1}\right),\left(x_{2}, r_{2}\right) \in \mathbb{Z} \times[0, \infty)$ and $b>a \geq \max \left(r_{1}, r_{2}\right)$, we have

$\mathbb{P}\left[\begin{array}{l}\eta_{s}^{\left(x_{1}, r_{1}\right)}, \eta_{s}^{\left(x_{2}, r_{2}\right)} \neq \Delta \text { for all } s \in[a, b], \\ \min \left(T^{\left(\eta_{s}^{\left(x_{1}, r_{1}\right)}, s\right)}, T^{\left(\eta_{s}^{\left(x_{2}, r_{2}\right)}, s\right)}\right)<\infty \text { for all } s \in[a, b]\end{array}\right]<e^{-c(b-a)}$.

(2) Let $\left(x_{1}, r_{1}\right),\left(x_{2}, r_{2}\right) \in \mathbb{Z} \times[0, \infty)$ and $\sigma$ be a stopping time (with respect to the sigma-algebra of Harris systems) with $\sigma \geq \max \left(r_{1}, r_{2}\right)$ almost surely. Let

$$
\tau=\inf \left\{t \geq \sigma: \eta_{t}^{\left(x_{1}, r_{1}\right)}, \eta_{t}^{\left(x_{2}, r_{2}\right)} \neq \Delta \text { and } T^{\left(\eta_{t}^{\left(x_{1}, r_{1}\right)}, t\right)}=T^{\left(\eta_{t}^{\left(x_{2}, r_{2}\right)}, t\right)}=\infty\right\} .
$$

For any $y_{1}, y_{2} \in \mathbb{Z}$ and events $E, F$ on Harris systems,

$$
\begin{aligned}
& \mathbb{P}\left[\tau<\infty, H_{[0, \tau]} \in E, \eta_{\tau}^{\left(x_{1}, r_{1}\right)}=y_{1}, \eta_{\tau}^{\left(x_{2}, r_{2}\right)}=y_{2} \text { and } H \circ \theta(0, \tau) \in F\right] \\
& =\mathbb{P}\left[\tau<\infty, H_{[0, \tau]} \in E, \eta_{\tau}^{\left(x_{1}, r_{1}\right)}=y_{1}, \eta_{\tau}^{\left(x_{2}, r_{2}\right)}=y_{2}\right] \cdot \tilde{\mathbb{P}}^{\left\{y_{1}, y_{2}\right\}}[H \in F] .
\end{aligned}
$$

Given $u>0$ and $t>0$, define $K^{u, t}=-13 u \sqrt{t}$ and the intervals

$$
\begin{aligned}
& I_{k}^{u, t}=K^{u, t}+((4 k-1) u \sqrt{t}, 4 k u \sqrt{t}), \quad 1 \leq k \leq 3 ; \\
& J_{0}^{u, t}=\left(-\infty, K^{u, t}\right] ; \\
& J_{k}^{u, t}=K^{u, t}+[(4 k-3) u \sqrt{t},(4 k-2) u \sqrt{t}], \quad 1 \leq k \leq 3 ; \\
& J_{4}^{u, t}=K^{u, t}+[13 u \sqrt{t}, \infty)=[0, \infty) .
\end{aligned}
$$

We will often omit the superscripts and write $K, I_{k}$ and $J_{k}$. These definitions, as well as the event treated in the following lemma, are illustrated in Figure 4.3.

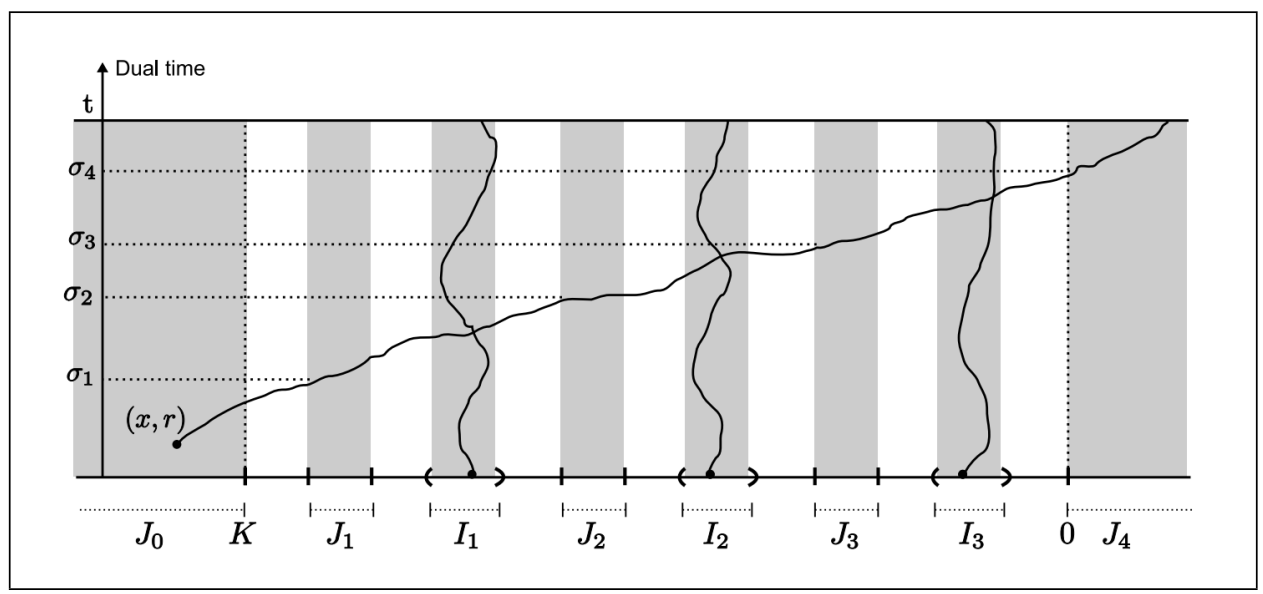

FiguRE 4.3. The event of Lemma 4.3.

Lemma 4.3. For any $u>0$, there exist $C=C(u)>0$ and $t_{0}=t_{0}(u)>0$ such that, for any $t \geq t_{0},(x, r) \in J_{0}^{u, t} \times[0, t], x_{1} \in I_{1}^{u, t}, x_{2} \in I_{2}^{u, t}$ and $x_{3} \in I_{3}^{u, t}$, we have $\mathbb{P}\left[\eta_{t}^{(x, r)} \geq 0\right.$ and $\eta_{s}^{x_{i}} \in I_{k}^{u, t}$ for all $k \in\{1,2,3\}$ and $\left.s \in[0, t]\right] \leq \frac{C}{t^{3 / 2}}$. 
Proof: Fix $u>0$. Choose $t$ large enough that

$$
2 t^{1 / 4}<u \sqrt{t}
$$

Fix $(x, r) \in J_{0} \times[0, t], x_{1} \in I_{1}, x_{2} \in I_{2}, x_{3} \in I_{3}$. Define

$$
\sigma_{k}=\inf \left\{s: \eta_{s}^{(x, r)} \geq \inf J_{k}\right\}, \quad 1 \leq k \leq 4
$$

The probability in the statement of the lemma is less than

$$
\mathbb{P}\left[\sigma_{4} \leq t \text { and } \eta_{s}^{x_{k}} \in I_{k} \text { for all } s \leq \sigma_{4} \text { and } k \leq 3\right]
$$

We will show that

$$
\begin{aligned}
\mathbb{P}\left[\sigma_{4} \leq\right. & \left.t, \eta_{s}^{x_{k}} \in I_{k} \text { for all } s \leq \sigma_{4} \text { and } k \leq 3\right] \\
& \leq C e^{-c t^{\gamma}}+\frac{C}{\sqrt{t}} \mathbb{P}\left[\sigma_{3} \leq t, \eta_{s}^{x_{k}} \in I_{k} \text { for all } k \leq 2 \text { and } s \leq \sigma_{3}\right]
\end{aligned}
$$

for some $c, C, \gamma$ that only depend on $u$. To this end, we first define the events

$$
\begin{aligned}
& E_{1}=\left\{\exists s, s^{\prime} \in[r, t]:\left|s-s^{\prime}\right|<t^{1 / 8}, \eta_{s}^{(x, r)} \neq \Delta, \eta_{s^{\prime}}^{(x, r)} \neq \Delta,\left|\eta_{s}^{(x, r)}-\eta_{s^{\prime}}^{(x, r)}\right|>t^{1 / 4}\right\}, \\
& E_{2}=\left\{\begin{array}{c}
\exists s, s^{\prime} \in[r, t]:\left|s-s^{\prime}\right| \geq t^{1 / 8}, \eta_{u}^{(x, r)} \neq \Delta, \eta_{u}^{x_{3}} \neq \Delta \\
\text { and } \min \left(T^{\left(\eta_{u}^{(x, r)}, u\right)}, T^{\left(\eta_{u}^{x_{3}}, u\right)}\right)<\infty \text { for all } u \in\left[s, s^{\prime}\right]
\end{array}\right\}
\end{aligned}
$$

and the random time

$$
\tau=\inf \left\{t \geq \sigma_{3}: \eta_{t}^{(x, r)} \neq \triangle, \eta_{t}^{x_{3}} \neq \triangle,\left(\eta_{t}^{(x, r)}, t\right) \leftrightarrow \infty,\left(\eta_{t}^{x_{3}}, t\right) \leftrightarrow \infty\right\}
$$

By (2.40) and (4.5), we can find $\gamma>0$ such that

$$
\mathbb{P}\left[E_{1} \cup E_{2}\right] \leq C e^{-c t^{\gamma}} .
$$

Using (4.6), we have

$$
\begin{aligned}
\left\{\sigma_{4} \leq t\right. & \left.; \eta_{s}^{x_{k}} \in I_{k} \text { for all } s \leq \sigma_{4}, k \leq 3\right\} \cap E_{1}^{c} \\
& \subseteq\left\{\begin{array}{l}
\sigma_{3} \leq t-t^{1 / 8} ; \eta_{s}^{x_{k}} \in I_{k} \text { for all } s \leq \sigma_{3} \text { and } k \leq 2 ; \\
\eta_{s}^{(x, r)} \leq \sup J_{3} \text { and } \eta_{s}^{x_{3}} \in I_{3} \text { for all } s \in\left[\sigma_{3}, \sigma_{3}+t^{1 / 8}\right] ; \\
\eta_{s}^{(x, r)}>\eta_{s}^{x_{3}}+u \sqrt{t} \text { for some } s \in\left[\sigma_{3}+t^{1 / 8}, t\right]
\end{array}\right\}
\end{aligned}
$$


We thus also get

$$
\begin{aligned}
& \left\{\sigma_{4} \leq t, \eta_{s}^{x_{k}} \in I_{k} \text { for all } s \leq \sigma_{4} \text { and } k \leq 3\right\} \cap E_{1}^{c} \cap E_{2}^{c} \\
& \subseteq\left\{\begin{array}{l}
\sigma_{3} \leq t-t^{1 / 8}, \eta_{s}^{x_{k}} \in I_{k} \text { for all } s \leq \sigma_{3} \text { and } k \leq 2, \\
\tau \leq \sigma_{3}+t^{1 / 8}, \eta_{\tau}^{(x, r)} \leq \sup J_{3}, \\
\eta_{\tau}^{x_{3}} \in I_{3}, \eta_{s}^{(x, r)}>\eta_{s}^{x_{3}}+u \sqrt{t} \text { for some } s \in[\tau, t]
\end{array}\right\} \\
& \subseteq\left\{\begin{array}{l}
\sigma_{3} \leq t, \eta_{s}^{x_{k}} \in I_{k} \text { for all } s \leq \sigma_{3} \text { and } k \leq 2, \\
\tau<\infty, \eta_{\tau}^{(x, r)} \leq \sup J_{3}, \eta_{\tau}^{x_{3}} \in I_{3}, \eta_{s}^{(x, r)}>\eta_{s}^{x_{3}}+u \sqrt{t} \text { for some } s \in[\tau, \tau+t]
\end{array}\right\} \\
& =\bigcup_{z \leq \sup J_{3}} \bigcup_{w \in I_{3}}\left\{\begin{array}{l}
\sigma_{3} \leq t, \eta_{s}^{x_{k}} \in I_{k} \text { for all } s \leq \sigma_{3} \text { and } k \leq 2, \\
\tau<\infty, \eta_{\tau}^{(x, r)}=z, \eta_{\tau}^{x_{3}}=w, \\
\eta_{s}^{(z, \tau)}>\eta_{s}^{(w, \tau)}+u \sqrt{t} \text { for some } s \in[\tau, \tau+t]
\end{array}\right\} \text {. }
\end{aligned}
$$

Using this set inclusion and (4.5) we obtain

$$
\begin{aligned}
& \mathbb{P}\left[\left\{\sigma_{4} \leq t, \eta_{s}^{x_{k}} \in I_{k} \text { for all } s \leq \sigma_{4}, k \leq 3\right\} \cap E_{1}^{c} \cap E_{2}^{c}\right] \\
& \leq \sum_{z \leq \sup J_{3}} \sum_{w \in I_{3}} \mathbb{P}\left[\begin{array}{c}
\sigma_{3} \leq t, \eta_{s}^{x_{k}} \in I_{k} \text { for all } s \leq \sigma_{3} \\
\text { and } k \leq 2, \tau<\infty, \eta_{\tau}^{(x, t)}=z, \eta_{\tau}^{x_{3}}=w
\end{array}\right] \\
& \cdot \tilde{\mathbb{P}}^{\{z, w\}}\left[\eta_{s}^{z}>\eta_{s}^{w}+u \sqrt{t} \text { for some } s \in[0, t]\right] \\
& \leq \mathbb{P}\left[\sigma_{3} \leq t, \eta_{s}^{x_{k}} \in I_{k} \text { for all } s \leq \sigma_{3} \text { and } k \leq 2\right] \\
& \cdot \sup _{z \leq \sup J_{3}, w \in I_{3}} \tilde{\mathbb{P}}^{\{z, w\}}\left[\eta_{s}^{z}>\eta_{s}^{w}+u \sqrt{t} \text { for some } s \in[0, t]\right] \\
& \stackrel{(2.53)}{\leq} \frac{C}{\sqrt{t}} \cdot \mathbb{P}\left[\sigma_{3} \leq t, \eta_{s}^{x_{k}} \in I_{k} \text { for all } s \leq \sigma_{3} \text { and } k \leq 2\right] .
\end{aligned}
$$

The desired result now follows from iterating this computation.

Lemma 4.4. For any $\varepsilon>0$ there exists $U>0$ such that, for large enough $t$,

$$
\mathbb{P}\left[\text { there exists }(x, r) \in\left[-U \sqrt{t}-t^{1 / 4},-U \sqrt{t}\right] \times\{0,1, \ldots,\lfloor t\rfloor\}: \eta_{t}^{(x, r)}>0\right]<\frac{\varepsilon}{U^{2}} \text {. }
$$

Proof: Let $N$ be a large integer to be chosen later. Define

$$
u=N^{2}, \quad U=13 u, \quad t>t_{0}(u), \text { where } t_{0}(u) \text { is as in Lemma 4.3. }
$$


For $k \in\{1,2,3\}$, let $\bar{I}_{k}^{u, t}$ be the set of $N$ sites (or $N+1$ sites, depending on parity) that are closest to the middle point of $I_{k}^{u, t}$. Define the events

$$
\begin{aligned}
& B_{1}=\left\{T^{\bar{I}_{k}^{u, t}}<\infty \text { for some } k \in\{1,2,3\}\right\}, \\
& B_{2}=\left\{\text { there exist } k \in\{1,2,3\}, y \in \bar{I}_{k}^{u, t}, s \leq t \text { such that } \eta_{s}^{y} \neq \triangle, \eta_{s}^{y} \notin I_{k}^{u, t}\right\}, \\
& B_{3}=\left\{\begin{array}{c}
\text { there exist }(x, r) \in\left[-U \sqrt{t}-t^{1 / 4},-U \sqrt{t}\right] \times\{0, \ldots,\lfloor t\rfloor\}, \\
y_{1} \in \bar{I}_{1}^{u, t}, y_{2} \in \bar{I}_{2}^{u, t}, y_{3} \in \bar{I}_{3}^{u, t} \text { such that } \\
\eta_{t}^{(x, r)}>0, \eta_{s}^{y_{i}} \in I_{i}^{u, t} \text { for each } i=1,2,3, s \leq t .
\end{array}\right\} .
\end{aligned}
$$

We then have

$\left\{\right.$ there exists $\left.(x, r) \in\left[-U \sqrt{t}-t^{1 / 4},-U \sqrt{t}\right] \times\{0,1, \ldots,\lfloor t\rfloor\}: \eta_{t}^{(x, r)}>0\right\} \subseteq \cup_{k=1}^{3} B_{k}$.

In what follows, $c$ and $C$ will denote constants that only depend on $\lambda$ and $R$ (recall that $\lambda$ and $R$ respectively denote the birth rate and range of the interactions of our process). Moreover, $C_{N}$ will denote constants that also depend on $N$. Of course, since $u=N^{2}$, constants that depend on $u$ also depend on $N$. We start bounding the probability of $B_{1}$ :

$$
\mathbb{P}\left[B_{1}\right] \leq \sum_{k=1}^{3} \mathbb{P}\left[T^{\bar{I}_{k}^{u, t}}<\infty\right] \stackrel{(2.10)}{\leq} \sum_{k=1}^{3} \exp \left\{c \cdot \# \bar{I}_{k}^{u, t}\right\} \leq 3 e^{-c N} .
$$

To bound the probability of $B_{2}$, fix $k \in\{1,2,3\}$ and $y \in \bar{I}_{k}^{u, t}$. As long as $t$ is large enough that $N<u \sqrt{t}$, we have

$$
\left\{\text { there exists } s \leq t: \eta_{s}^{y} \notin I_{k}^{u, t}\right\} \subseteq\left\{\sup _{0 \leq s \leq t}\left|\eta_{s}^{y}-y\right|>u \sqrt{t} / 2\right\}
$$

and, by (2.40) and (2.43), the probability of this event is less than $C e^{-c \frac{u^{2} t}{4 t}}+$ $C t e^{-c \frac{u \sqrt{t}}{2}}$ for some $c, C>0$. We thus get

$$
\mathbb{P}\left[B_{2}\right] \leq 3 N\left(C e^{-c \frac{u^{2} t}{4 t}}+C t e^{-c \frac{u \sqrt{t}}{2}}\right)=3 N\left(C e^{-c \frac{N^{4}}{4}}+C t e^{-c \frac{N^{2} \sqrt{t}}{2}}\right) .
$$

We now turn to $B_{3}$. Note that there are at most $t^{5 / 4}$ candidates for $(x, r)$ and $N^{3}$ candidates for $y_{1}, y_{2}, y_{3}$. Using Lemma 4.3, there exists $C_{N}>0$ such that

$$
\mathbb{P}\left[B_{3}\right] \leq N^{3} \cdot t^{5 / 4} \cdot \frac{C_{N}}{t^{3 / 2}} \leq \frac{C_{N} \cdot N^{3}}{t^{1 / 4}} .
$$

Putting these bounds together and rearranging constants, we get

$$
\begin{gathered}
U^{2} \cdot \mathbb{P}\left[\text { there exists }(x, r) \in\left[-U \sqrt{t}-t^{1 / 4},-U \sqrt{t}\right] \times\{0,1, \ldots,\lfloor t\rfloor\}: \eta_{t}^{(x, r)}>0\right] \\
\leq C N^{4} e^{-c N}+C N^{5} e^{-c N^{4}}+C t N^{5} e^{-c N^{2} \sqrt{t}}+\frac{C_{N} N^{7}}{t^{\frac{1}{4}}} .
\end{gathered}
$$

Now, given $\varepsilon>0$, we first choose $N^{*}$ such that the sum of the first two terms on (4.10) is less than $\varepsilon / 2$. Next, we choose $t^{*}>t_{0}\left(\left(N^{*}\right)^{2}\right)$ such that, for $N^{*}$ and any $t>t^{*}$, the sum of the third and fourth terms in (4.10) is less than $\varepsilon / 2$. This completes the proof of the lemma, with $U=13\left(N^{*}\right)^{2}$. 
Lemma 4.5. For any $\varepsilon>0$ there exists $U>0$ such that, for large enough $t$,

$$
\mathbb{P}\left[\left|r_{s}\right|,\left|\ell_{s}\right| \leq U \sqrt{t} \text { for all } s \leq t\right]>1-\frac{\varepsilon}{U^{2}} .
$$

Proof: Given $\varepsilon>0$, we will find $U>0$ such that

$$
\mathbb{P}\left[\inf _{0 \leq s \leq t} \ell_{s}<-U \sqrt{t}-t^{1 / 4}\right]<\frac{\varepsilon}{U^{2}} \text { if } t \text { is large enough }
$$

and

$$
\mathbb{P}\left[\inf _{0 \leq s \leq t} \ell_{s} \geq-U \sqrt{t}-t^{1 / 4}, \inf _{0 \leq s \leq t} r_{s}<-2 U \sqrt{t}\right] \stackrel{t \rightarrow \infty}{\longrightarrow} 0
$$

the statement of the lemma clearly follows from these statements and symmetry.

For (4.11), we remark that, using the joint construction of the multitype contact process and the ancestor processes, (4.9) can be rewritten as

$$
\mathbb{P}\left[\text { there exists }(x, r) \in\left[-U \sqrt{t}-t^{1 / 4},-U \sqrt{t}\right] \times\{0,1, \ldots,\lfloor t\rfloor\}: \xi_{r}^{h}(x)=2\right]<\frac{\varepsilon}{U^{2}} .
$$

Letting $A_{t}$ be the event that appears in the above probability, we also have

$$
\begin{aligned}
& \mathbb{P}\left[A_{t}^{c} \cap\left\{\inf _{0 \leq s \leq t} \ell_{s}<-U \sqrt{t}-t^{1 / 4}\right\}\right] \\
& \leq \sum_{r=0}^{\lfloor t\rfloor} \mathbb{P}\left[[-U \sqrt{t}, \infty) \times\{r\} \leftrightarrow\left(-\infty,-U \sqrt{t}-t^{1 / 4}\right] \times[r, r+1]\right] \leq t e^{-c t^{1 / 4}}
\end{aligned}
$$

by a comparison with a Poisson random variable. (4.11) is thus proved.

We now turn to (4.12). If the event inside the probability occurs, then we have $\ell_{s} \geq-U \sqrt{t}-t^{1 / 4}$ for all $s \in[0, t]$ and $r_{s^{*}}<-2 U \sqrt{t}$ for some $s^{*} \in[0, t]$. This gives

$$
r_{s *}=\sup \left\{x: \xi_{s^{*}}^{h}(x)=1\right\}<-2 U \sqrt{t}, \quad \ell_{s^{*}}=\inf \left\{x: \xi_{s^{*}}^{h}(x)=2\right\} \geq-U \sqrt{t}-t^{1 / 4}
$$

$$
\Longrightarrow \xi_{s^{*}}^{h}(y)=0 \text { for all } y \in\left[-2 U \sqrt{t},-U \sqrt{t}-t^{1 / 4}\right) \text {. }
$$

This gives

$$
\begin{aligned}
& \mathbb{P}\left[\inf _{0 \leq s \leq t} \ell_{s} \geq-U \sqrt{t}-t^{1 / 4}, \inf _{0 \leq s \leq t} r_{s}<-2 U \sqrt{t}\right] \\
& \leq \mathbb{P}\left[\text { there exists } s \in[0, t] \text { such that } \xi_{s}^{h} \equiv 0 \text { on }\left[-2 U \sqrt{t},-U \sqrt{t}-t^{1 / 4}\right)\right] .
\end{aligned}
$$

The probability on the right-hand side can be bounded from above similarly to how we proceeded in Lemma 2.3, so that (4.12) follows.

Proof of Lemma 4.1 We recall the definition of $i_{s}^{t}$ in (2.58). Given $\varepsilon>0$, using the previous lemma we choose $U$ so that

$$
\mathbb{P}\left[\sup _{0 \leq s \leq t}\left|i_{s}\right| \leq \frac{U}{2} \sqrt{t}\right]>1-\frac{\varepsilon}{2 U^{2}} .
$$

Using Theorem 2.14, we choose $K$ so that, for any $r>0$,

$$
\mathbb{P}\left[\left|i_{s}-i_{s}^{r}\right| \leq K \text { for all } s \geq r\right]>1-\frac{\varepsilon}{2 U^{2}} .
$$


Then, if $t$ is large enough that $U \sqrt{t}>2 K$ we have, for any $r>0$,

$$
\begin{aligned}
& \mathbb{P}\left[\sup _{r \leq s \leq r+t}\left|i_{s}-i_{r}\right|>U \sqrt{t}\right] \\
& \leq \mathbb{P}\left[\sup _{r \leq s \leq r+t}\left|i_{s}-i_{s}^{r}\right|>\frac{U}{2} \sqrt{t}\right]+\mathbb{P}\left[\sup _{r \leq s \leq r+t}\left|i_{s}^{r}-i_{r}\right|>\frac{U}{2} \sqrt{t}\right] \\
& \leq \mathbb{P}\left[\sup _{r \leq s \leq r+t}\left|i_{s}-i_{s}^{r}\right|>K\right]+\mathbb{P}\left[\sup _{0 \leq s \leq t}\left|i_{s}\right|>\frac{U}{2} \sqrt{t}\right]<\frac{\varepsilon}{U^{2}} .
\end{aligned}
$$

\section{Interface regeneration}

In this section we will prove Theorem 2.14. We will often consider multitype contact processes with different initial configurations simultaneously. When we do so, we always assume that all these processes are constructed on the same probability space, using a single Harris system $H$.

We start defining some classes of subsets of the space of configurations $\{0,1,2\}^{\mathbb{Z}}$. Recall the definition of $\Omega \subset\{0,1,2\}^{\mathbb{Z}}$ in (2.54). Define

$\Gamma_{S, L}=\left\{\begin{array}{ll}\xi \in \Omega: & \text { there exist } a<b \text { with } b-a \leq L, r(\xi), \ell(\xi) \in(a, b), \\ \xi \equiv 1 \text { on }[a-S, a] \text { and } \xi \equiv 2 \text { on }[b, b+S]\end{array}\right\}, S, L>0$.

The homogeneously and fully occupied intervals $[a-S, a],[b, b+S]$ that appear in the above definition will be referred to as "isolation segments". The reason is that we think of them as isolating the interface (which is contained in $(a, b)$ ) from the "outside" $[a-S, b+S]^{c}$, so that, if $S$ is large, we can hope that the configuration in the outside never has any effect on the evolution of the interface.

Our second class of configurations will depend on a preliminary definition. Given $\xi_{0} \in \Omega$, let

$$
\tilde{\xi}_{0}=\mathbb{1}_{\left(-\infty,\left\lfloor i\left(\xi_{0}\right)\right\rfloor\right]}+2 \cdot \mathbb{1}_{\left(\left\lfloor i\left(\xi_{0}\right)\right\rfloor, \infty\right)} .
$$

Also let $\left(\xi_{t}\right)$ and $\left(\tilde{\xi}_{t}\right)$ be contact processes started from $\xi_{0}$ and $\tilde{\xi}_{0}$, respectively (constructed with the same Harris system). We now let

$$
\Omega_{\varepsilon, K}=\left\{\xi_{0} \in \Omega: \mathbb{P}\left[\left|i\left(\xi_{t}\right)-i\left(\tilde{\xi}_{t}\right)\right|<K \text { for all } t\right]>1-\varepsilon\right\} .
$$

We will separately prove the following two propositions:

Proposition 5.1. (Large isolation segments allow for regeneration). For any $\varepsilon>0$ there exists $S>0$ such that the following holds. For any $L>0$ there exists $K=K(\varepsilon, S, L)>0$ such that $\Gamma_{S, L} \subseteq \Omega_{\varepsilon, K}$.

Proposition 5.2. (Large isolation segments are found not too far). For any $\varepsilon>0$ and $S>0$ there exists $L>0$ such that, for any $t \geq 0$,

$$
\mathbb{P}\left[\xi_{t}^{h} \in \Gamma_{S, L}\right]>1-\varepsilon .
$$


Proof of Theorem 2.14. Fix $\varepsilon>0$. Choose $S=S(\varepsilon)$ as in Proposition 5.1, then choose $L=L(\varepsilon, S)$ as in Proposition 5.2, and finally choose $K=K(\varepsilon, S, L)$ as in Proposition 5.1. Now, for any $t \geq 0$ we have

$$
\mathbb{P}\left[\xi_{t}^{h} \in \Omega_{\varepsilon, K}\right] \geq \mathbb{P}\left[\xi_{t}^{h} \in \Gamma_{S, L}\right]>1-\varepsilon .
$$

Now, for any $s \geq 0$ we have

$$
\mathbb{P}\left[\sup _{t \geq s}\left|i_{t}^{s}-i_{t}\right|>K\right] \leq \mathbb{P}\left[\xi_{s}^{h} \notin \Omega_{\varepsilon, K}\right]+\mathbb{P}\left[\sup _{t \geq s}\left|i_{t}^{s}-i_{t}\right|>K \mid \xi_{s}^{h} \in \Omega_{\varepsilon, K}\right] .
$$

The first probability on the right-hand side is less than $\varepsilon$ by (5.3) and the second one is less than $\varepsilon$ by the definition of $\Omega_{\varepsilon, K}$.

5.1. Proof of Proposition 5.1. In proving Proposition 5.1, we will need to argue that, if $S$ is large and $\xi_{0} \in \Omega$ is a configuration such that, for some $a, b, L$,

$$
a<r\left(\xi_{0}\right), \ell\left(\xi_{0}\right)<b<a+L, \quad \xi_{0} \equiv 1 \text { on }[a-S, a], \quad \xi_{0} \equiv 2 \text { on }[b, b+S],
$$

then the interface of $\left(\xi_{t}\right)$ is with high probability never too far from that of the process started from $\tilde{\xi}_{0}$, the "heaviside version" of $\xi_{0}$.

The comparison between the interfaces of $\left(\xi_{t}\right)$ and $\left(\tilde{\xi}_{t}\right)$ will be indirect. We will define an intermediate process $\left(\hat{\xi}_{t}\right)$, started from what we call an "almost-heaviside version" of $\xi_{0}$ :

$$
\hat{\xi}_{0}=\mathbb{1}_{(-\infty, a]}(x)+\mathbb{1}_{(a, b)}(x) \cdot \xi_{0}(x)+2 \cdot \mathbb{1}_{[b, \infty)}(x) .
$$

We will then have two comparison results, Lemma 5.3 and Lemma 5.4, the former allowing us to compare the interfaces of $\left(\tilde{\xi}_{t}\right)$ and $\left(\hat{\xi}_{t}\right)$, and the latter allowing us to compare the interfaces of $\left(\hat{\xi}_{t}\right)$ and $\left(\xi_{t}\right)$.

We now give the full statements of our comparison lemmas. Here and in the rest of this section, $\bar{\beta}$ is the constant of Lemma 2.1.

Lemma 5.3. (Comparison I: Processes with initial configurations which are equal and non-empty at all but finitely many sites). For any $\varepsilon>0$ and $L>0$ there exists $t_{0}>0$ such that the following holds. If $I$ is an interval of length at most $L$ and $\hat{\xi}_{0}, \hat{\xi}_{0} \in\{0,1,2\}^{\mathbb{Z}}$ are such that $\hat{\xi}_{0}(x)=\hat{\xi}_{0}(x) \neq 0$ for all $x \in \mathbb{Z} \backslash I$, then

$$
\mathbb{P}\left[\hat{\xi}_{t}=\hat{\hat{\xi}}_{t} \text { for all } t \geq t_{0}\right]>1-\varepsilon \text {. }
$$

Lemma 5.4. (Comparison II: Process started from configuration $\xi_{0}$ with isolation segments compared to process started from almost-heaviside version of $\left.\xi_{0}\right)$. For any $\varepsilon>0$ there exists $S_{0}>0$ such that the following holds. Assume $S \geq S_{0}$ and $\xi_{0}$ satisfies, for some $a, b \in \mathbb{Z}$ with $a<0<b$ :

$$
\begin{aligned}
& \xi_{0} \equiv 1 \text { on }[a-S, a] ; \\
& a<r\left(\xi_{0}\right), \ell\left(\xi_{0}\right)<b ; \\
& \xi_{0} \equiv 2 \text { on }[b, b+S] .
\end{aligned}
$$

Let $\left(\hat{\xi}_{t}\right)_{t \geq 0}$ be the process started from

$$
\hat{\xi}_{0}(x)=\mathbb{1}_{(-\infty, a]}(x)+\mathbb{1}_{(a, b)}(x) \cdot \xi_{0}(x)+2 \cdot \mathbb{1}_{[b, \infty)}(x) .
$$


Then, with probability larger than $1-\varepsilon$,

$$
\begin{array}{ll}
\text { for every } t \geq 0, \quad r\left(\xi_{t}\right)=r\left(\hat{\xi}_{t}\right), \quad \ell\left(\xi_{t}\right)=\ell\left(\hat{\xi}_{t}\right) \text { and } \\
& a-\frac{S}{2}-\bar{\beta} t<r\left(\xi_{t}\right), \ell\left(\xi_{t}\right)<b+\frac{S}{2}+\bar{\beta} t .
\end{array}
$$

The proofs of the comparison lemmas will be done later in this section; first we will show how they give Proposition 5.1. The argument is sketched in Figure 5.4.

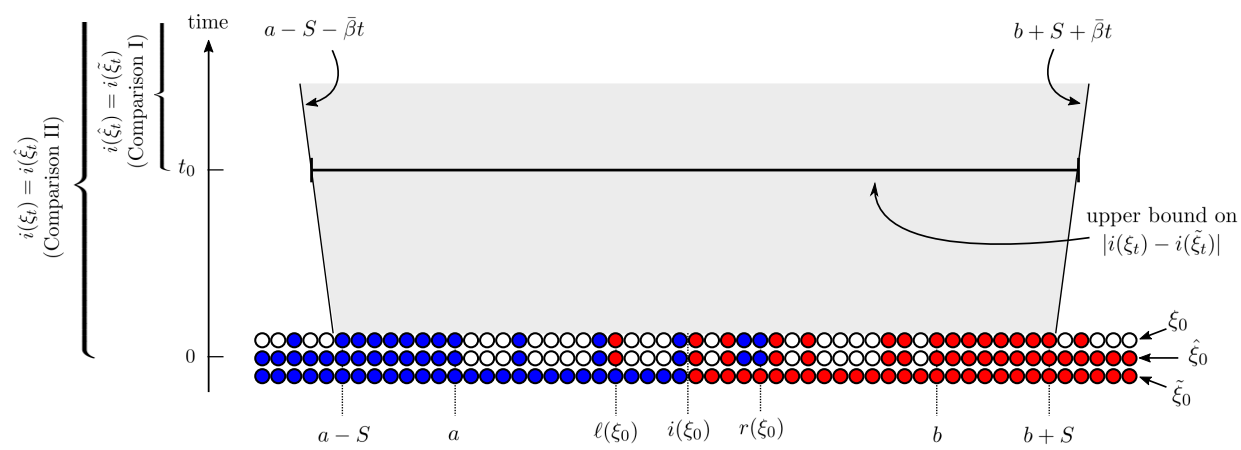

Figure 5.4. Sketch of the argument for Proposition 5.1. With high probability, (1.) the interfaces of the three processes, $\xi, \hat{\xi}$ and $\tilde{\xi}$ are contained in the grey zone at all times, (2.) $i\left(\xi_{t}\right)=i\left(\hat{\xi}_{t}\right)$ for all $t$ and (3.) $i\left(\hat{\xi}_{t}\right)=i\left(\tilde{\xi}_{t}\right)$ for all $t \geq t_{0}$. If all these hold, then $\left|i\left(\xi_{t}\right)-i\left(\tilde{\xi}_{t}\right)\right| \leq b+S+\bar{\beta} t_{0}-\left(a-S-\bar{\beta} t_{0}\right)$ for all $t$.

Proof of Proposition 5.1. Given $\varepsilon>0$, we choose $S$ large enough corresponding to $\varepsilon / 3$ in Lemma 5.4. Increasing $S$ if necessary, by Lemma 2.13, we can also assume the following (recall that $r_{t}=r\left(\xi_{t}^{h}\right)$ and $\ell_{t}=\ell\left(\xi_{t}^{h}\right)$, where $\left(\xi_{t}^{h}\right)$ is the process started from the heaviside configuration).

$$
\mathbb{P}\left[r_{t}, \ell_{t} \in[S-\bar{\beta} t, S+\bar{\beta} t] \text { for all } t \geq 0\right]>1-\varepsilon .
$$

Then, given $L>0$, we choose $t_{0}$ corresponding to $\varepsilon / 3$ and $L$ in Lemma 5.3.

Now assume $\xi_{0} \in \Gamma_{S, L}$. Then, there exist $a<b$ as prescribed in (5.1); note in particular that $r\left(\xi_{0}\right), \ell\left(\xi_{0}\right) \in(a, b)$, so that $i\left(\xi_{0}\right) \in(a, b)$. Let

$$
\begin{aligned}
& \hat{\xi}_{0}(x)=\mathbb{1}_{(-\infty, a]}(x)+\mathbb{1}_{(a, b)}(x) \cdot \xi_{0}(x)+2 \cdot \mathbb{1}_{[b, \infty)}(x), \\
& \tilde{\xi}_{0}(x)=\mathbb{1}_{\left(-\infty,\left\lfloor i\left(\xi_{0}\right)\right\rfloor\right]}+2 \cdot \mathbb{1}_{\left(\left\lfloor i\left(\xi_{0}\right)\right\rfloor, \infty\right)}
\end{aligned}
$$

and $\left(\hat{\xi}_{t}\right),\left(\tilde{\xi}_{t}\right)$ be the processes started from these configurations. By our choice of $S$ and $t_{0}$, with probability larger than $1-\varepsilon$ the following three events occur:

for all $t \geq 0, i\left(\xi_{t}\right)=i\left(\hat{\xi}_{t}\right) \in[a-S-\bar{\beta} t, b+S+\bar{\beta} t]$;

for all $t \geq 0, i\left(\tilde{\xi}_{t}\right) \in\left[\left\lfloor i\left(\xi_{0}\right)\right\rfloor-S-\bar{\beta} t,\left\lfloor i\left(\xi_{0}\right)\right\rfloor+S+\bar{\beta} t\right] \subset[a-S-\bar{\beta} t, b+S+\bar{\beta} t]$;

for all $t \geq t_{0}, i\left(\hat{\xi}_{t}\right)=i\left(\tilde{\xi}_{t}\right)$. 
If these events all occur, we have

$$
\begin{aligned}
& \left|i\left(\xi_{t}\right)-i\left(\tilde{\xi}_{t}\right)\right| \leq b-a+2 S+2 \bar{\beta} t_{0} \leq L+2 S+2 \bar{\beta} t_{0} \text { if } t \leq t_{0} \text { and } \\
& \left|i\left(\xi_{t}\right)-i\left(\tilde{\xi}_{t}\right)\right|=0 \text { if } t>t_{0} .
\end{aligned}
$$

The desired result now holds for $K=L+2 S+2 \bar{\beta} t_{0}$.

Let us now prove our first comparison lemma. The proof is a simple application of the convergence to zero of the density of the set of all first ancestor processes, given by equation (2.52).

Proof of Lemma 5.3. Since $\left(\hat{\xi}_{t}\right)$ and $\left(\hat{\hat{\xi}}_{t}\right)$ are constructed from the same Harris system $H$, it suffices to find $t_{0}$ such that

$$
\mathbb{P}\left[\hat{\xi}_{t_{0}}=\hat{\hat{\xi}}_{t_{0}}\right]>1-\varepsilon .
$$

For a fixed $t_{0}>0$, consider the system of first ancestor processes $\left(\left(\eta_{t}^{x}\right)_{0 \leq t \leq t_{0}}: x \in\right.$ $\mathbb{Z})$ constructed from the time-reversed Harris system $\hat{H}_{\left[0, t_{0}\right]}$. Since $\hat{\xi}_{0} \equiv \overline{\hat{\xi}}_{0}$ on $\mathbb{Z} \backslash I$, we have

$$
\begin{aligned}
\mathbb{P}\left[\hat{\xi}_{t_{0}}=\hat{\hat{\xi}}_{t_{0}}\right] \stackrel{(2.25)}{\geq} \mathbb{P}\left[\hat{\xi}_{0}\left(\eta_{t_{0}}^{x}\right)=\hat{\hat{\xi}}_{0}\left(\eta_{t_{0}}^{x}\right) \neq 0 \text { for all } x \in \mathbb{Z} \text { with } \eta_{t_{0}}^{x} \neq \Delta\right] \\
\geq \mathbb{P}\left[\eta_{t_{0}}^{x} \notin I \text { for all } x \in \mathbb{Z}\right] \geq 1-(\# I) \cdot \mathbb{P}\left[0 \in\left\{\eta_{t_{0}}^{x}: x \in \mathbb{Z}\right\}\right] .
\end{aligned}
$$

The result now follows from taking $t_{0}$ large enough, depending on $\varepsilon$ and $L$, by (2.52).

The proof of the second comparison lemma, Lemma 5.4, will depend on a onesided version of that statement, which we now state and prove.

Lemma 5.5. For any $\varepsilon>0$ there exists $S_{0}>0$ such that the following holds for any $S \geq S_{0}$. Assume $\xi_{0}$ satisfies:

$$
\begin{aligned}
& \xi_{0} \equiv 2 \text { on }[0, S] ; \\
& r\left(\xi_{0}\right)<0 .
\end{aligned}
$$

Let $\left(\xi_{t}^{\prime}\right)_{t \geq 0}$ be the process started from

$$
\xi_{0}^{\prime}(x)=\mathbb{1}_{(-\infty, 0)}(x) \cdot \xi_{0}(x)+2 \cdot \mathbb{1}_{[0, \infty)}(x) .
$$

Then, with probability larger than $1-\varepsilon$ we have

$$
\begin{array}{ll}
\text { for any } t \geq 0, & \xi_{t} \equiv \xi_{t}^{\prime} \text { on }(-\infty, S / 2+\beta t], \\
& \ell\left(\xi_{t}\right)=\ell\left(\xi_{t}^{\prime}\right), r\left(\xi_{t}\right)=r\left(\xi_{t}^{\prime}\right) \text { and } \\
& \ell\left(\xi_{t}\right), r\left(\xi_{t}\right)<S / 2+\bar{\beta} t .
\end{array}
$$

Proof: Given $S>0$, we write

$$
L_{t}^{(1)}=\frac{S}{4}+\frac{\bar{\beta}}{2} t, \quad L_{t}^{(2)}=\frac{S}{2}+\bar{\beta} t, \quad t \geq 0
$$


Fix $\varepsilon>0$. By Lemmas 2.1, 2.3 and 2.13, if $S$ is large enough, then with probability larger than $1-\varepsilon$ all the following three events occur:

$$
\begin{aligned}
& E_{1}=\left\{\text { for all }(x, t) \text { with } x \leq L_{t}^{(2)}, \xi_{t}(x)=0 \text { if and only if } \xi_{t}^{\prime}(x)=0\right\}, \\
& E_{2}=\left\{\text { for all } t \geq 0, \text { there exists } x \text { such that } L_{t}^{(1)} \leq x \leq L_{t}^{(2)} \text { and } \xi_{t}^{\prime}(x) \neq 0\right\}, \\
& E_{3}=\left\{r\left(\xi_{t}^{\prime}\right)<L_{t}^{(1)} \text { for all } t \geq 0\right\} .
\end{aligned}
$$

We will also assume that $S>4 R$.

We will now state and prove two auxiliary claims.

Claim 1. On $E_{1} \cap E_{3},\left\{x: \xi_{t}(x)=1\right\}=\left\{x: \xi_{t}^{\prime}(x)=1\right\}$ and $\left\{x: \xi_{t}(x)=2\right\} \subseteq\{x:$ $\left.\xi_{t}^{\prime}(x)=2\right\}$ for all $t$.

To see that this holds, first note that

$$
\left\{x: \xi_{0}(x)=1\right\}=\left\{x: \xi_{0}^{\prime}(x)=1\right\},\left\{x: \xi_{0}(x)=2\right\} \subseteq\left\{x: \xi_{0}^{\prime}(x)=2\right\},
$$

so applying (2.19) we get

$$
\left\{x: \xi_{t}(x)=1\right\} \supseteq\left\{x: \xi_{t}^{\prime}(x)=1\right\},\left\{x: \xi_{t}(x)=2\right\} \subseteq\left\{x: \xi_{t}^{\prime}(x)=2\right\} \text { for all } t .
$$

Let us prove that, under the assumption that $E_{1} \cap E_{3}$ occurs, the first inclusion is in fact an equality. Fix $(x, t)$ such that $\xi_{t}(x)=1$. By Lemma 2.4, there exists an infection path $\gamma$ such that $\xi_{0}(\gamma(0))=1, \gamma(t)=x$ and $\gamma$ only jumps to space-time points that are unoccupied in $\left(\xi_{s}\right)_{s \geq 0}$, that is, $\gamma$ satisfies (2.13). By Lemma 2.5, we also have

$$
\xi_{s}(\gamma(s))=1, \quad s \in[0, t] .
$$

Let $x_{0}=\gamma(0), x_{1}, \ldots, x_{n}=x$ be the successive positions of $\gamma$; also let $t_{0}=0$ and $0<t_{1}<\cdots<t_{n} \leq t$ be the jump times of $\gamma$, so that:

$\gamma \equiv x_{0}$ on $\left[0, t_{1}\right), \gamma \equiv x_{1}$ on $\left[t_{1}, t_{2}\right), \cdots, \gamma \equiv x_{n-1}$ on $\left[t_{n-1}, t_{n}\right), \gamma \equiv x$ on $\left[t_{n}, t\right]$.

We will prove by induction that, for each $i$, we have

$$
\xi_{t_{i}}^{\prime}\left(x_{i}\right)=1
$$

the statement of Claim 1 will immediately follow. The statement of (5.15) is clear for $i=0$. Assume that it holds for some $i<n$. Since $\gamma$ is an infection path, there is no recovery mark in $\left[t_{i}, t_{i+1}\right)$, hence it follows from the induction hypothesis that

$$
\xi_{t_{i+1}-}^{\prime}\left(x_{i}\right)=1 \text {. }
$$

Since $\gamma$ jumps from $x_{i}$ to $x_{i+1}$ at time $t_{i+1}$, using (2.13) we have

$$
\xi_{t_{i+1}-}\left(x_{i+1}\right)=0 \text {. }
$$

Now note that

$$
x_{i+1} \leq x_{i}+R \leq r\left(\xi_{t_{i}}^{\prime}\right)+R \leq L_{t_{i}}^{(1)}+R \leq L_{t_{i+1}}^{(1)}+R \leq L_{t_{i+1}}^{(2)},
$$

where the second inequality follows from the induction hypothesis and the third inequality follows from our assumption that $E_{3}$ occurs. Using (5.17) and (5.18) together with our assumption that $E_{1}$ occurs, we get

$$
\xi_{t_{i+1}-}^{\prime}\left(x_{i+1}\right)=0 \text {. }
$$

Finally, putting (5.16) and (5.19) we conclude that $\xi_{t_{i+1}}^{\prime}\left(x_{i+1}\right)=1$ completing the proof by induction. 
Claim 2. On $E_{2} \cap E_{3}, \ell\left(\xi_{t}^{\prime}\right) \leq L_{t}^{(2)}$ for all $t$.

Indeed, by the definition of $E_{2}$, for any $t$ there exists $x \in\left[L_{t}^{(1)}, L_{t}^{(2)}\right]$ such that $\xi_{t}^{\prime}(x) \neq 0$, and by the definition of $E_{3}$ we have $r\left(\xi_{t}^{\prime}\right)<L_{t}^{(1)}$, so that $x>r\left(\xi_{t}^{\prime}\right)$, thus $\xi_{t}^{\prime}(x)=2$, thus $\ell\left(\xi_{t}^{\prime}\right) \leq x \leq L_{t}^{(2)}$.

We are now ready to conclude. From Claim 1 and the definition of $E_{1}$, we have that

$$
\text { on } E_{1} \cap E_{3}, \xi_{t}(x)=\xi_{t}^{\prime}(x) \text { for all } t \geq 0, x \leq L_{t}^{(2)} .
$$

From Claim 1 and the definition of $E_{3}$,

$$
\text { on } E_{1} \cap E_{3}, r\left(\xi_{t}\right)=r\left(\xi_{t}^{\prime}\right)<L_{t}^{(1)}<L_{t}^{(2)} .
$$

From Claim 1 and Claim 2,

$$
\text { on } E_{1} \cap E_{2} \cap E_{3}, \ell\left(\xi_{t}\right)=\ell\left(\xi_{t}^{\prime}\right)<L_{t}^{(2)} \text {. }
$$

Proof of Lemma 5.4. Let $\left(\xi_{t}\right)$ and $\left(\hat{\xi}_{t}\right)$ be as in the statement of the lemma. We will also need $\left(\xi_{t}^{\prime}\right)_{t \geq 0}$, the process started from

$$
\xi_{0}^{\prime}(x)=\mathbb{1}_{(-\infty, b)}(x) \cdot \xi_{0}(x)+2 \cdot \mathbb{1}_{[b, \infty)}(x) .
$$

Given $\varepsilon>0$, by Lemma 5.5, $S_{0}$ can be chosen so that, if (5.5) and (5.6) hold, then

$$
\mathbb{P}\left[\begin{array}{ll}
\text { for every } t \geq 0, & \ell\left(\xi_{t}\right)=\ell\left(\xi_{t}^{\prime}\right), r\left(\xi_{t}\right)=r\left(\xi_{t}^{\prime}\right) \\
& \text { and } \ell\left(\xi_{t}\right), r\left(\xi_{t}\right)<b+\frac{S}{2}+\beta t
\end{array}\right]>1-\varepsilon / 2 .
$$

Now, note that (5.6) and the definition of $\xi_{0}^{\prime}$ imply

$$
\xi_{0}^{\prime} \equiv 1 \text { on }[a-S, a], \quad r\left(\xi_{0}^{\prime}\right)>a,
$$

so that we can again use Lemma 5.5 (and symmetry) to obtain that

$$
\mathbb{P}\left[\begin{array}{ll}
\text { for any } t \geq 0, & \ell\left(\xi_{t}^{\prime}\right)=\ell\left(\hat{\xi}_{t}\right), r\left(\xi_{t}^{\prime}\right)=r\left(\hat{\xi}_{t}\right) \\
& \text { and } \ell\left(\xi_{t}^{\prime}\right), r\left(\xi_{t}^{\prime}\right)>a-\frac{S}{2}-\beta t
\end{array}\right]>1-\varepsilon / 2 .
$$

Putting (5.20) and (5.21) together, we obtain the desired result.

5.2. Proof of Proposition 5.2. Recall the definition of $\Omega$ in (2.54). Given $\xi \in \Omega$, we write

$$
m(\xi)=\min \{r(\xi), \ell(\xi)\}, \quad M(\xi)=\max \{r(\xi), \ell(\xi)\},
$$

so that $\xi(x) \in\{0,1\}$ for any $x<m(\xi)$ and $\xi(x) \in\{0,2\}$ for any $x>M(\xi)$. Also let

$$
m_{t}=m\left(\xi_{t}^{h}\right)=\min \left\{r_{t}, \ell_{t}\right\}, \quad M_{t}=M\left(\xi_{t}^{h}\right)=\max \left\{r_{t}, \ell_{t}\right\} .
$$

Our proof of Proposition 5.2 will consist of three main steps. First, we will argue that the proposition follows from the weaker result:

Lemma 5.6. For $S, L>0$, let

$\Gamma_{S, L}^{\prime}=\{\xi \in \Omega$ : there exists $b \in(M(\xi), M(\xi)+L]$ such that $\xi(x) \equiv 2$ on $[b, b+S]\}$.

For any $\varepsilon>0$ and $S>0$ there exists $L>0$ such that, for any $t \geq 0$,

$$
\mathbb{P}\left[\xi_{t}^{h} \in \Gamma_{S, L}^{\prime}\right]>1-\varepsilon .
$$


(Note that $\Gamma_{S, L}^{\prime} \supset \Gamma_{S, L}$, so Lemma 5.6 is indeed weaker than Proposition 5.2). Second, we will prove that Lemma 5.6 follows from the yet weaker result:

Lemma 5.7. For $k, L \in \mathbb{Z}_{+}, k<L$, let

$$
\Pi_{k, L}=\{\xi \in \Omega: \#\{x \in(M(\xi), M(\xi)+L]: \xi(x)=2\} \geq k\} .
$$

For any $\varepsilon>0$ and $k \in \mathbb{Z}_{+}$there exists $L>0$ such that, for any $t \geq 0$,

$$
\mathbb{P}\left[\xi_{t}^{h} \in \Pi_{k, L}\right]>1-\varepsilon .
$$

(Note that $\Pi_{S, L+S} \supset \Gamma_{S, L}^{\prime}$, so Lemma 5.7 is indeed weaker than Lemma 5.6). Third, we will use Theorem 1.1 to prove Lemma 5.7.

5.2.1. Step 1: proof of Proposition 5.2 assuming Lemma 5.6. Our first step is an easy application of tightness of the interface size and symmetry.

Proof of Proposition 5.2. Fix $S>0$ and $\varepsilon>0$. Using (1.7), choose $L_{0}>0$ such that

$$
\mathbb{P}\left[M_{t}-m_{t} \leq L_{0}\right]>1-\varepsilon / 3 \text { for all } t \geq 0
$$

Using Lemma 5.6, choose $L_{1}>0$ such that

$$
\mathbb{P}\left[\xi_{t}^{h} \in \Gamma_{S, L_{1} / 3}^{\prime}\right]>1-\varepsilon / 3 \text { for all } t \geq 0 .
$$

Since the set of configurations $\Gamma_{S, L_{1} / 3}^{\prime}$ is increasing with $L_{1}$, we may assume that $L_{1} / 3>L_{0}$. Using symmetry, we also have

$$
\mathbb{P}\left[\begin{array}{c}
\text { there exists } a \in\left[m_{t}-L_{1} / 3, m_{t}\right) \\
\text { such that } \xi_{t}^{h} \equiv 1 \text { on }[a-S, a]
\end{array}\right]>1-\varepsilon / 3 \text { for all } t \geq 0 .
$$

Now note that if for $t \geq 0$ the events inside the probabilities in (5.23), (5.24) and (5.25) all occur, then $\xi_{t}^{h} \in \Gamma_{S, L_{1}}$.

5.2.2. Step 2: proof of Lemma 5.6 assuming Lemma 5.7. Apart from Lemma 5.7, we will need to use the following claim, which shows how, when $L$ and $k$ are appropriately chosen, depending on $S$, configurations in $\Pi_{k, L}$ can lead to configurations in $\Gamma_{S, L}^{\prime}$ with high probability:

Claim 5.8. For any $\varepsilon>0$ and $S>0$ there exist $\tau>0$ and $k \in \mathbb{Z}_{+}$such that, for any $L \geq k$,

$$
\xi_{0} \in \Pi_{k, L} \quad \Longrightarrow \quad \mathbb{P}\left[\xi_{\tau} \in \Gamma_{S, L}^{\prime}\right]>1-\varepsilon .
$$

Before proving this claim, let us show how to establish Lemma 5.6.

Proof of Lemma 5.6. Fix $\varepsilon$ and $S$. We first choose $\tau$ and $k$ corresponding to $\varepsilon / 2$ and $S$ in Claim 5.8, and then choose $L$ corresponding to $\varepsilon / 2$ and $k$ in Lemma 5.7 (we may assume $L \geq k$ ). We then have, for any $t \geq \tau$,

$\mathbb{P}\left[\xi_{t}^{h} \in \Gamma_{S, L}^{\prime}\right] \geq \mathbb{P}\left[\xi_{t}^{h} \in \Gamma_{S, L}^{\prime} \mid \xi_{t-\tau}^{h} \in \Pi_{k, L}\right] \cdot \mathbb{P}\left[\xi_{t-\tau}^{h} \in \Pi_{k, L}\right] \geq(1-\varepsilon / 2)^{2}>1-\varepsilon$.

That is, (5.22) holds for all $t \geq \tau$. It is then easy to show that we can increase $L$ if necessary so that the result also holds for $t \in[0, \tau)$.

We now turn to the proof of Claim 5.8. The idea of the proof is as follows. If $\tau$ is small (depending on $\varepsilon$ ), $k$ is large (depending on $\varepsilon, S$ and $\tau$ ), $L \geq k$ and $\xi_{0} \in \Pi_{k, L}$, then the following will be shown to hold with probability larger than $1-\varepsilon$ : 
- $M\left(\xi_{s}\right)$ will be constant for $s \in[0, \tau]$ (that is, the right extremity of the interface will not change in this time interval);

- one of the (at least $k$ ) sites $x \in\left(M\left(\xi_{0}\right), M\left(\xi_{0}\right)+L\right]$ with $\xi_{0}(x)=2$ will "germinate", by time $\tau$, an isolating interval of length $S$. This means simply that $x$ will produce enough descendants to fully occupy the interval $[x, x+S]$.

This will produce $\xi_{\tau} \in \Gamma_{S, L}^{\prime}$. We now proceed to the actual proof.

Proof of Claim 5.8. Given $x \in \mathbb{Z}$ and $\tau>0$, define the freezing event $A_{\tau}(x)$ as the event that the space-time set $[x-R, x+R] \times[0, \tau]$ has no recovery mark and no start or endpoint of any transmission arrow. The reason we call this a freezing event is the following:

$$
\text { if } \xi_{0} \in \Omega \text { and } A_{\tau}\left(M\left(\xi_{0}\right)\right) \text { occurs, then } M\left(\xi_{s}\right)=M\left(\xi_{0}\right) \text { for all } s \in[0, \tau] \text {, }
$$

that is, the right extremity of the interface is frozen in this time interval (a similar statement holds for the left extremity). This can be easily verified by inspecting the two possible cases $M\left(\xi_{0}\right)=r\left(\xi_{0}\right)$ and $M\left(\xi_{0}\right)=\ell\left(\xi_{0}\right)$.

Given $x \in \mathbb{Z}, \tau>0$ and $S>0$, define the germination event $B_{\tau, S}(x)$ as the event that

- for all $y \in[x, x+S]$, there is an infection path from $(x, 0)$ to $(y, \tau)$ which is entirely contained in $[x, x+S]$;

- there is no arrow originating from $[x, x+S]^{c} \times[0, \tau]$ and entering $[x, x+$ $S] \times[0, \tau]$.

If this event occurs, then $x$ germinates a fully occupied interval:

$$
\text { if } \xi_{0} \in \Omega, x>M\left(\xi_{0}\right) \text { and } B_{\tau, S}(x) \text { occurs, then } \xi_{\tau} \equiv 2 \text { on }[x, x+S] \text {. }
$$

It is worth observing that, by translation invariance of the Harris system, the probabilities of $A_{\tau}(x)$ and $B_{\tau, S}(x)$ do not depend on $x$. Additionally, by making prescriptions on the Poisson processes of the Harris system in a finite space-time region, it can be seen that

$$
\mathbb{P}\left[B_{\tau, S}(0)\right]=: \delta_{\tau, S}>0 \text { for all } \tau, S>0 .
$$

Fix $\varepsilon>0$ and $S>0$. We choose $\tau>0$ small enough that

$$
\mathbb{P}\left[A_{\tau}(0)\right]>1-\varepsilon / 2 .
$$

We then choose $k \in \mathbb{Z}_{+}$so that

$$
k^{\prime}:=\lfloor k /(2 R+S+1)\rfloor \text { satisfies }\left(1-\delta_{\tau, S}\right)^{k^{\prime}}<\varepsilon / 2
$$

(note in particular that $k \geq 2 R+S+1$ ).

Now that $\tau$ and $k$ have been chosen, assume that $L \geq k$ and fix $\xi_{0} \in \Pi_{k, L}$. By the definition of $\Pi_{k, L}$, we can find

$$
\begin{aligned}
& x_{1}, \ldots, x_{k^{\prime}} \in\left(M\left(\xi_{0}\right)+2 R+S+1, M\left(\xi_{0}\right)+L\right] \text { with } \quad x_{i+1}>x_{i}+2 R+S+1 \\
& \text { and } \xi_{0}\left(x_{i}\right)=2 \text { for all } i \text {. }
\end{aligned}
$$

We then have

$$
\mathbb{P}\left[\xi_{1} \in \Gamma_{S, L}^{\prime}\right] \stackrel{(5.1),(5.26),(5.27)}{\geq} \mathbb{P}\left[A_{\tau}\left(M\left(\xi_{0}\right)\right) \cap\left(\cup_{i=1}^{k} B_{\tau, S}\left(x_{i}\right)\right)\right] .
$$


Since the intervals $\left[M\left(\xi_{0}\right)-R, M\left(\xi_{0}\right)+R\right]$ and $\left\{\left[x_{i}-R, x_{i}+R+S\right], 1 \leq i \leq\right.$ $\left.k^{\prime}\right\}$ are all disjoint, the events in the probability on the right-hand side are all independent. Hence, using (5.28), (5.29) and (5.30), this probability is larger than $1-\frac{\varepsilon}{2}-\left(1-\delta_{S}\right)^{k^{\prime}}>1-\varepsilon$.

5.2.3. Step 3: proof of Lemma 5.7. For $t \geq 0$, define

$X_{t}^{(0)}=M_{t}=\max \left\{r\left(\xi_{t}^{h}\right), \ell\left(\xi_{t}^{h}\right)\right\}, X_{t}^{(j+1)}=\min \left\{x>X_{t}^{(j)}: \xi_{t}^{h}(x) \neq 0\right\}, j=0,1, \ldots$

We remark that the minimum that defines each $X_{t}^{(j)}, j \geq 1$, is well defined since at any time there are infinitely many occupied sites to the right of the origin. An equivalent formulation of Lemma 5.7 is the statement that, for all $k \in \mathbb{Z}_{+}$, the process $\left(X_{t}^{(k)}-M_{t}\right)_{t \geq 0}$ is stochastically tight, that is,

for all $\varepsilon>0$ there exists $L>0$ such that $\mathbb{P}\left[X_{t}^{(k)}-M_{t}>L\right]<\varepsilon$ for all $t \geq 0$.

Our proof will be by contradiction. We will assume that (5.32) fails for some $k$ and will show that this would imply that, for a deterministic sequence $\left(t_{n}\right)$, the interface size $M_{t_{n}}-m_{t_{n}}$ is larger than $n$ with non-vanishing probability. We will then argue that this contradicts Theorem 1.1.

Proof of Lemma 5.7. Suppose the statement is false. Then, there exist $k \in \mathbb{Z}_{+}$and $\varepsilon>0$ such that, for all $L>0$, there exists $t_{L}>0$ such that $\mathbb{P}\left[X_{t_{L}}^{(k)}-M_{t_{L}}>L\right] \geq \varepsilon$. In particular, by letting $L$ vary along the natural numbers, we obtain a sequence $\left(t_{n}\right)$ such that

$$
\mathbb{P}\left[X_{t_{n}}^{(k)}-M_{t_{n}}>n\right] \geq \varepsilon \text { for all } n \in \mathbb{Z}_{+} .
$$

By tightness of the size of the interface (as given by (1.7)), we can then find $L_{0}>0$ such that

$$
\mathbb{P}\left[M_{t_{n}}-m_{t_{n}} \leq L_{0}, X_{t_{n}}^{(k)}-M_{t_{n}}>n\right]>\varepsilon / 2 \text { for all } n \in \mathbb{Z}_{+} .
$$

We will now show that (5.33) implies that one time unit after $t_{n}$, the interface has probability at least $\frac{\varepsilon}{2} \cdot \exp \left\{(-1-2 R \lambda)\left(2 R+L_{0}+k\right)\right\}$ of consisting of a gap of size at least $n$ (that is, $\ell_{t_{n}+1} \geq r_{t_{n}+1}+n$ ). The idea for this argument is essentially contained in Figure 5.5. The fact that the mentioned lower bound $\frac{\varepsilon}{2} \cdot \exp \{(-1-$ $\left.2 R \lambda)\left(2 R+L_{0}+k\right)\right\}$ does not depend on $n$ will then be used to yield a contradiction with tightness of the interface size, (1.7).

Let us denote by $E_{n}$ the event inside the probability on the left-hand side of (5.33). Also define the event

$$
F_{n}=E_{n} \cap\left\{\begin{array}{c}
\text { in the time interval }\left[t_{n}, t_{n}+1\right], \text { all vertices in } \\
{\left[m_{t_{n}}-R, M_{t_{n}}\right] \cup\left\{X_{t_{n}}^{(1)}, \ldots, X_{t_{n}}^{(k)}\right\} \cup\left(X_{t_{n}}^{(k)}, X_{t_{n}}^{(k)}+R\right]} \\
\text { have a death mark and do not originate any arrow. }
\end{array}\right\}
$$

See Figure 5.5 for an illustration of this definition. Since the set of vertices that appears in the definition of $F_{n}$ contains at most $2 R+L_{0}+k$ vertices, we have $\mathbb{P}\left[F_{n} \mid E_{n}\right]=\exp \left\{(-1-2 R \lambda)\left(2 R+L_{0}+k\right)\right\}$, so that, by (5.33),

$$
\mathbb{P}\left[F_{n}\right] \geq \frac{\varepsilon}{2} \cdot \exp \left\{(-1-2 R \lambda)\left(2 R+L_{0}+k\right)\right\} .
$$




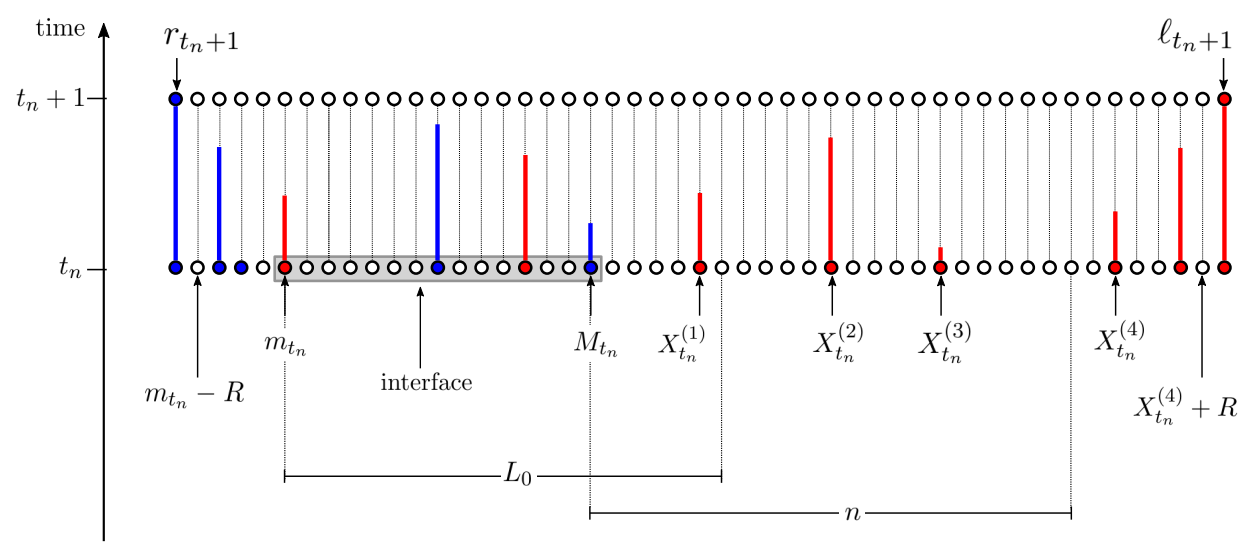

FiguRE 5.5. Illustration of the events $E_{n}$ and $F_{n}$ with $k=4$ (additional parameters in this picture are: $R=4, L_{0}=20$ and $n=22$ ). The event $E_{n}$ occurs because $M_{t_{n}}<m_{t_{n}}+L_{0}$ and $X_{t_{n}}^{(k)}=X_{t_{n}}^{(4)}>M_{t_{n}}+n$. The event $F_{n}$ occurs because in addition, all occupants of sites in $\left[m_{t_{n}}-R, X_{t_{n}}^{(k)}+R\right]$ at time $t_{n}$ die within one second without giving birth. As can be seen, this implies that the interface consists of a gap of size larger than $n$ at time $t_{n}+1$.

Additionally,

$$
F_{n} \subseteq\left\{r_{t_{n}+1}<m_{t_{n}}, \ell_{t_{n}+1}>M_{t_{n}}+n\right\} \subseteq\left\{\ell_{t_{n}+1}-r_{t_{n}+1}>n\right\} .
$$

Now, (5.34) and (5.35) together imply

$$
\mathbb{P}\left[\ell_{t_{n}+1}-r_{t_{n}+1}>n\right]>\frac{\varepsilon}{2} \cdot \exp \left\{(-1-2 R \lambda)\left(2 R+L_{0}+k\right)\right\} \text { for all } n .
$$

On the other hand, (1.7) implies that there exists $L^{\prime}$ such that

$$
\mathbb{P}\left[\left|\ell_{t}-r_{t}\right|>L^{\prime}\right]<\frac{\varepsilon}{2} \cdot \exp \left\{(-1-2 R \lambda)\left(2 R+L_{0}+k\right)\right\} \quad \text { for all } t \geq 0 .
$$

Since (5.36) and (5.37) contradict each other, the proof is complete.

\section{Acknowledgements}

We would like to thank the referee of ALEA, whose detailed report led us to a substantial improvement of the clarity of exposition of the paper.

\section{References}

E. Andjel, T. Mountford, L. P. R. Pimentel and D. Valesin. Tightness for the interface of the one-dimensional contact process. Bernoulli 16 (4), 909-925 (2010). MR2759162. 
P. Billingsley. Convergence of probability measures. Wiley Series in Probability and Statistics: Probability and Statistics. John Wiley \& Sons, Inc., New York, second edition (1999). ISBN 0-471-19745-9. A Wiley-Interscience Publication. MR1700749.

T. E. Harris. Contact interactions on a lattice. Ann. Probability 2, 969-988 (1974). MR0356292.

G. F. Lawler and V. Limic. Random walk: a modern introduction, volume 123 of Cambridge Studies in Advanced Mathematics. Cambridge University Press, Cambridge (2010). ISBN 978-0-521-51918-2. MR2677157.

T. M. Liggett. Stochastic interacting systems: contact, voter and exclusion processes, volume 324 of Grundlehren der Mathematischen Wissenschaften [Fundamental Principles of Mathematical Sciences]. Springer-Verlag, Berlin (1999). ISBN 3-540-65995-1. MR1717346.

C. Neuhauser. Ergodic theorems for the multitype contact process. Probab. Theory Related Fields 91 (3-4), 467-506 (1992). MR1151806.

R. Sun. Convergence of coalescing nonsimple random walks to the Brownian Web. Ph.D. thesis, New York University (2005). MR2706830.

D. Valesin. Multitype contact process on $\mathbb{Z}$ : extinction and interface. Electron. J. Probab. 15, no. 73, 2220-2260 (2010). MR2748404. 